\title{
Withaferin A Improves Nonalcoholic Steatohepatitis in Mice ${ }^{[\mathrm{s}}$
}

\author{
Daxesh P. Patel, ${ }^{1}$ Tingting Yan, ${ }^{1}$ Donghwan Kim, Henrique B. Dias, Kristopher W. Krausz, \\ Shioko Kimura, and Frank J. Gonzalez
}

\begin{abstract}
Laboratory of Metabolism, Center for Cancer Research, National Cancer Institute, National Institutes of Health, Bethesda, Maryland (D.P.P., T.Y., D.K., H.B.D., K.W.K., S.K., F.J.G.) and Laboratory of Cellular Biophysics and Inflammation, Pontifical Catholic University of Rio Grande do Sul, Rio Grande do Sul, Brazil (H.B.D.)
\end{abstract}

Received January 30, 2019; accepted August 13, 2019

\begin{abstract}
Nonalcoholic steatohepatitis (NASH) is the progressive stage of nonalcoholic fatty liver disease that highly increases the risk of cirrhosis and liver cancer, and there are few therapeutic options available in the clinic. Withaferin A (WA), extracted from the ayurvedic medicine Withania somnifera, has a wide range of pharmacological activities; however, little is known about its effects on NASH. To explore the role of WA in treating $\mathrm{NASH}$, two well defined NASH models were used, the methionine-choline-deficient diet and the $40 \mathrm{kcal} \%$ high-fat diet (HFD). In both NASH models, WA treatment or control vehicle was administered to evaluate its hepatoprotective effects. As assessed by biochemical and histologic analyses, WA prevented and therapeutically improved liver injury in both
\end{abstract}

models, as revealed by lower serum aminotransaminases, hepatic steatosis, liver inflammation, and fibrosis. In the HFD-induced NASH model, both elevated serum ceramides and increased hepatic oxidative stress were decreased in the WA-treated group compared with the control vehicle-treated group. To further explore whether WA has an anti-NASH effect independent of its known action in leptin signaling associated with obesity, leptin signaling-deficient ob/ob mice maintained on an HFD were used to induce NASH. WA therapeutically reduced $\mathrm{NASH}$ in HFD-treated leptin-deficient ob/ob mice, thus demonstrating a leptin-independent hepatoprotective effect. This study revealed that WA treatment could be an option for NASH treatment.

\section{Introduction}

Nonalcoholic steatohepatitis (NASH) is the progressive stage of nonalcoholic fatty liver disease (NAFLD) that results in a high risk of the end-stage liver diseases, cirrhosis and hepatocellular cancer (Farrell and Larter, 2006; Michelotti et al., 2013). While virus-induced hepatitis has been sharply reduced with vaccine application and curative drugs (Scott et al., 2015; Flemming et al., 2017), there continues to be an increase in NAFLD incidence due to the rapid rise of obesity and diabetes (Wong et al., 2014; Estes et al., 2018). NASH is currently listed as the second leading cause of liver disease among adults awaiting liver transplantation in the United States (Wong et al., 2015) and is estimated to overtake hepatitis $\mathrm{C}$ virus infection as the leading cause of liver transplantation in the United States in the coming decades

This research was supported by the Intramural Research Program of the National Institutes of Health [National Cancer Institute, Center for Cancer Research]; H.B.D. was supported by a fellowship from the CAPES Foundation, Ministry of Education of Brazil, Brazil.

No conflicts of interest, financial or otherwise, are declared by the authors.

${ }^{1}$ D.P.P. and T.Y. contributed equally to this study.

https://doi.org/10.1124/jpet.119.256792.

S This article has supplemental material available at jpet.aspetjournals.org.
(Oseini and Sanyal, 2017). Although some conventional therapies, such as vitamin $\mathrm{E}$ and pioglitazone, could improve steatosis and inflammation, few treatments that could significantly decrease fibrosis, one of the strongest indicators of liver damage caused by NASH, have been found (Cassidy and Syed, 2016; Oseini and Sanyal, 2017), indicating that while market-available drugs are mostly effective in treating hepatic steatosis, they have minimal effects on fibrosis associated with NASH. There are no current Food and Drug Administrationapproved therapies for treating NASH, and the development of novel medical treatments is urgently needed.

Withaferin A (WA) is a steroidal lactone derived from the plant Withania somnifera used in traditional ayurvedic medicine (Vanden Berghe et al., 2012). WA was originally used as an antitumor agent (Lee and Choi, 2016) and has other pharmacological properties, including cardioprotective, antiinflammation, and antioxidant activities (Vanden Berghe et al., 2012). Recently, WA was demonstrated in mice to be a leptin sensitizer with strong antidiabetic properties that could decrease obesity-associated metabolic abnormalities, including hepatic steatosis (Lee et al., 2016). Several other studies demonstrated that WA has hepatoprotective activity as revealed by its ability to mitigate acetaminophen-induced

ABBREVIATIONS: Acer, alkaline ceramidase; ALT, alanine aminotransferase; AST, aspartate aminotransferase; AUC, area under the plasma drug concentration-time curve; Cers, ceramide synthase; ER, endoplasmic reticulum; HFD, high-fat-diet; IL, interleukin; LFD, low-fat-diet; MCD, methionine-choline-deficient; MCS, methionine-choline-sufficient; NAFLD, nonalcoholic fatty liver disease; NASH, nonalcoholic steatohepatitis; NEFA, nonesterified fatty acid; NLRP3, NLR family pyrin domain containing 3; NRF2, nuclear factor erythroid 2-related factor 2; Sgms, sphingomyelin synthase; Smpd, sphingomyelin phosphodiesterase; Sptlc, serine palmitoyltransferase, long-chain base subunit; Srebp1c, sterol regulatory element-binding protein 1c; TC, total cholesterol; TG, triglyceride; WA, withaferin A. 
A

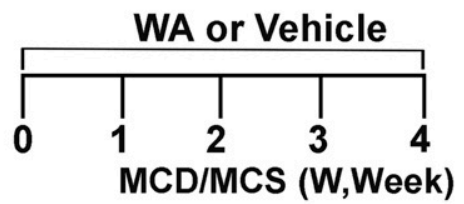

B

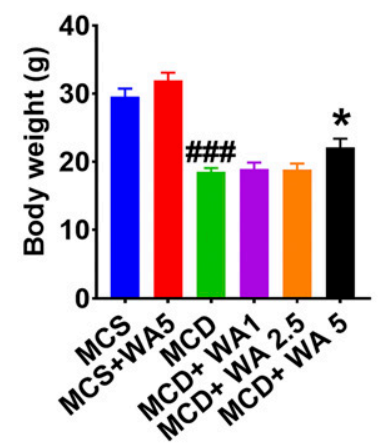

C
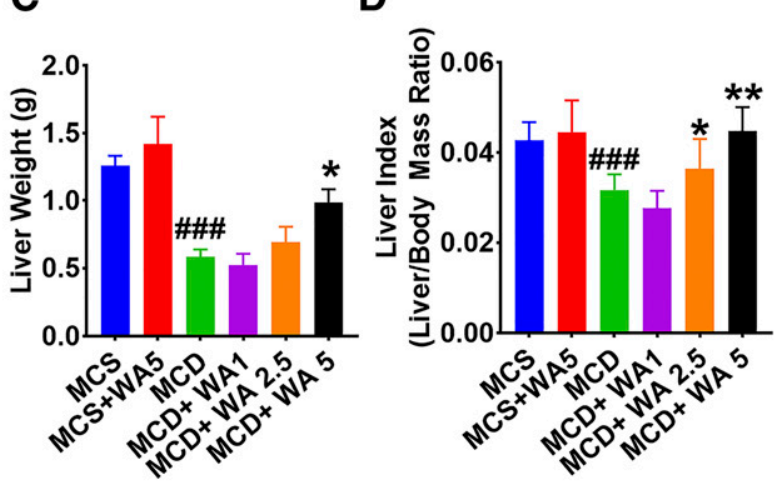

Fig. 1. Effect of WA on body weight, liver weight, liver index, and liver histology in MCS diet-fed mice. (A) Experiment scheme for testing the preventive effects of WA in the MCD-induced liver injury model. (B) Effect of WA on body weight. (C) Effect of WA on liver weight. (D) Effect of WA on liver index. (E) Effect of WA on $\mathrm{H} \& \mathrm{E}$ staining of MCS diet-fed mouse livers. Scale bar, $100 \mu \mathrm{m}$. Data were presented as means \pm S.D. MCS, mice fed the MCS diet and treated with vehicle; MCS+WA5, mice fed the MCS diet and treated with $5 \mathrm{mg} / \mathrm{kg}$ of WA only; MCD, mice fed the MCD diet and treated with vehicle; MCD+WA1, mice fed the MCD diet and treated with $1 \mathrm{mg} / \mathrm{kg}$ of WA; MCD+WA2.5, mice fed the MCD diet and treated with $2.5 \mathrm{mg} / \mathrm{kg}$ of WA; MCD + WA5, mice fed the MCD diet and treated with $5 \mathrm{mg} / \mathrm{kg}$ of WA. $n=5$ mice per group. Statistical differences were determined by the two-tailed $t$ test between two groups or by one-way ANOVA among multiple MCD diet-fed groups. ${ }^{\# \# \#} P<0.005$ when compared with MCS group; $* P<0.05 ; * * P<0.01$ when compared with the MCD group.

E

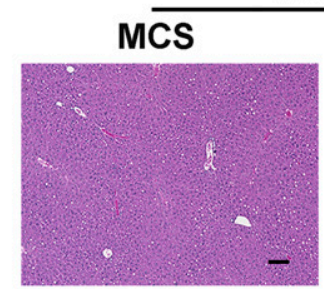

H\&E

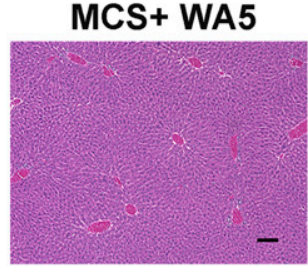

acute liver injury (Jadeja et al., 2015; Palliyaguru et al., 2016) and alleviate bromobenzene-induced liver injury (Vedi and Sabina, 2016). Since obesity is usually associated with insulin resistance and fatty liver, the pharmacological potential of WA in treating NASH needs to be evaluated.

NAFLD/NASH is known to be caused by a hepatic overload of fatty acids, leading to the production of toxic lipids that could cause hepatic oxidative stress, inflammation, endoplasmic reticulum (ER) stress, and cell death (Friedman et al., 2018). Among the toxic lipids, ceramides are signaling molecules that accumulate in the blood and tissues in animal models of metabolic diseases. High cellular levels of ceramides are correlated with inflammation, cell death, oxidative stress, ER stress, and insulin resistance (Pagadala et al., 2012; Chaurasia and Summers, 2015). Thus, strategies that decrease ceramides could efficiently improve NAFLD and NASH (Kurek et al., 2014; Jiang et al., 2015; Xie et al., 2017). On the other hand, WA is known to suppress oxidative stress, evidenced by its nuclear factor erythroid 2-related factor 2 (NRF2)-dependent effect in alleviating acetaminophen-induced liver injury and its effect in inducing heme oxygenase expression in endothelial cells via activating the NRF2 pathway (Vanden Berghe et al., 2012; Jadeja et al., 2015; Heyninck et al., 2016; Palliyaguru et al., 2016). In addition, WA is a known leptin sensitizer that could both leptin signaling-dependently and leptin signalingindependently reduce diet-induced obesity as reported previously (Lee et al., 2016). Although there is no conclusive evidence that leptin is of value for the treatment of NAFLD patients, leptin was suggested to have a potential role in NASH treatment, as revealed by the fact that recombinant leptin administration showed a beneficial effect in hepatic steatosis in NAFLD patients with hyperleptinemia (Polyzos et al., 2015) or lipodystrophy patients (Javor et al., 2005). However, whether WA modulates ceramide homeostasis and alleviates oxidative stress when treating NASH and whether WA affects NASH dependent on its effect on leptin signaling are still not known.

In the present study, two widely-used NASH models, the methionine-choline-deficient (MCD) diet and the high-fat diet (HFD), were first used to evaluate the efficacy of WA in treating NASH. WA had both preventive and therapeutic effects in improving NASH, as revealed by significant decreases of serum aminotransferases, hepatic steatosis, inflammation, ER stress, and fibrosis. In the HFD-induced NASH model, WA alleviated NASH-associated oxidative stress and lowered serum ceramide levels. Using leptin-deficient ob/ob mice maintained on a NASH-promoting HFD, WA had a therapeutic effect in improving HFD-induced NASH in ob/ob mice, suggesting a leptin-independent anti-NASH effect of WA. The present results indicate that WA could be repurposed as a novel therapy for treating NASH patients.

\section{Materials and Methods}

Chemicals and Reagents. WA was purchased from ChromaDex (Irvine, CA). PeroxiDetect kit, superoxide dismutase kit, glutathione assay kit, and dimethylsulfoxide were purchased from Sigma-Aldrich (St. Louis, MO). Alanine aminotransferase (ALT) and aspartate aminotransferase (AST) kits were purchased from Catachem (Oxford, CT). Total cholesterol (TC), triglyceride (TG), and nonesterified fatty acid (NEFAs) kits were purchased from Wako Pure Chemical Industries (Osaka, Japan). Ceramide standards C16, C18, C20, C22, C24, C18:1, 



Fig. 2. WA dose-dependently alleviated MCD diet-induced liver injury. (A and B) Analysis of serum ALT (A) and AST (B) levels. (C and D) Analysis of liver TC (C) and TG (D) levels. (E and F) Analysis of serum TC (E) and TG (F) levels. (G) Analysis of hepatic proinflammatory cytokine mRNA levels. (H) H\&E staining of livers from MCD diet-fed mice treated with vehicle or $5 \mathrm{mg} / \mathrm{kg}$ WA. (I) Oil Red O staining of livers from MCD diet-fed mice treated with vehicle or $5 \mathrm{mg} / \mathrm{kg}$ WA. Scale bar, $100 \mu \mathrm{m}$. Data were presented as means \pm S.D. Group descriptions for the MCS, MCS + WA5, MCD, MCD + WA1, MCD+WA2.5, and MCD+WA5 groups were the same as described in the legend to Fig. $1 . n=5$ mice per group. ${ }^{\#} P<0.05 ;{ }^{\# \#} P<0.01 ;{ }^{\# \# \#} P<0.005$ when compared with the MCS group; $* P<0.05 ; * * P<0.01$; $* * * P<0.005$ when compared with the MCD group. Statistical differences were determined by the two-tailed $t$ test or one-way ANOVA. Tnfa, tumor necrosis factor $\alpha$.

and C24:1 were purchased from Avanti Polar Lipids (Alabaster, AL). Rodent diets with $40 \mathrm{kcal} \%$ high fat, $20 \mathrm{kcal} \%$ fructose, and $2 \%$ cholesterol (HFD, catalog number D09100301 for C57BL/6N mice and catalog number D09100310 for ob/ob mice) and $10 \mathrm{kcal} \%$ fat control diet [low-fat diet (LFD), catalog number D09100304] were purchased from Research Diets (New Brunswick, NJ). Methionine-choline-sufficient (MCS) diet and MCD diet were purchased from Dyets Inc. (Bethlehem, PA).

Drug Preparation and Dosing. The WA stock solution was dissolved in dimethylsulfoxide and frozen, and before use, it was diluted with saline to generate the treatment doses (the final dimethylsulfoxide percentage was the same for all final injection solutions). WA was injected intraperitoneally $(0.1 \mathrm{ml} / 20$-g mouse $)$ at the doses indicated once per day before the dark cycle of the day.

Animal Studies. Age-matched 6- to 8-week-old C57BL/6N males were housed in a specific pathogen-free environment controlled for temperature and light $\left(25^{\circ} \mathrm{C}, 12\right.$-hour light/dark cycle $)$ and humidity
(45\%-65\%). The National Cancer Institute Animal Care and Use Committee approved all animal experiments conducted in this study. The mice were administered WA at doses of $1,2.5$, and $5 \mathrm{mg} / \mathrm{kg}$ for the MCD model and at a dose of $5 \mathrm{mg} / \mathrm{kg}$ for the HFD models. To test the preventative effect of WA in treating MCD-induced NASH, the mice were randomly divided into six groups: 1) MCS: mice fed the MCS diet and treated with control vehicle; 2) MCS+WA5: mice fed the MCS diet and treated with $5 \mathrm{mg} / \mathrm{kg}$ WA; 3) MCD: mice fed the MCD diet and treated with control vehicle; 4) MCD+WA1: mice fed the MCD diet and treated with $1 \mathrm{mg} / \mathrm{kg} \mathrm{WA}$; 5) MCD+WA2.5: mice fed the MCD diet and treated with $2.5 \mathrm{mg} / \mathrm{kg}$ WA; and 6) MCD+WA5: mice fed the MCD diet and treated with $5 \mathrm{mg} / \mathrm{kg}$ WA. All mice were fed the MCS or MCD diets for 4 weeks, and WA or control vehicle was injected once per day from the first day of diet feeding. To test the therapeutic effect of WA in treating MCD diet-induced NASH, the mice were fed the MCD diet for 6 weeks and then injected with WA once a day at the dose of $5 \mathrm{mg} / \mathrm{kg}$ 
A
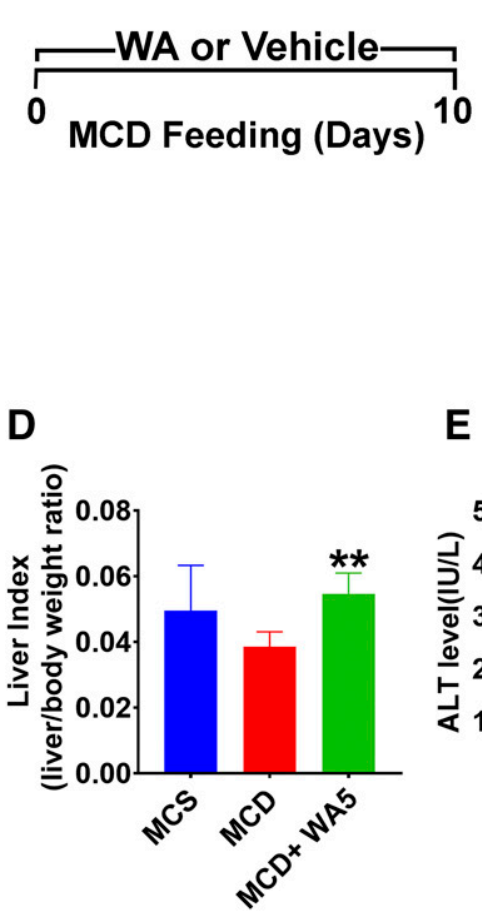

E

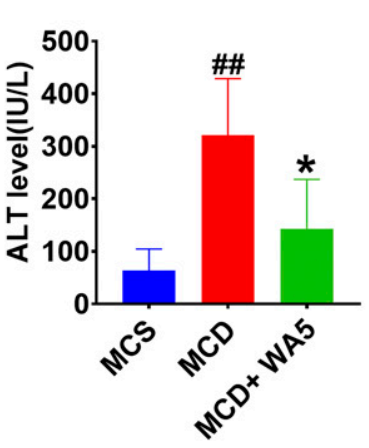

C

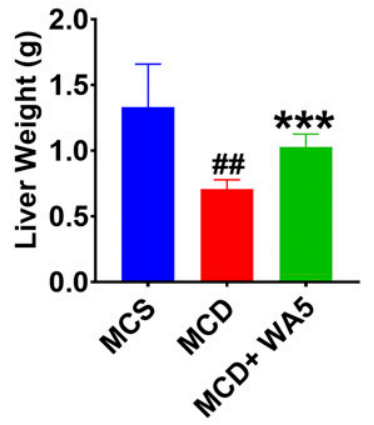

Fig. 3. WA alleviated MCD diet-induced liver injury independent of body weight change. (A) Experiment scheme for testing the therapeutic effect of WA in MCD diet-induced NASH model. (B) Body weights. (C) Liver weights. (D) Liver indexes calculated as the ratio of liver weight to body weight. (E) Serum ALT levels. (F) Serum AST levels. Data were presented as means \pm S.D. Group descriptions for MCS, $\mathrm{MCD}$, and MCD+WA5 were the same as described in the legend to Fig. $1 . n=5$ mice per group. Data were presented as means \pm S.D. Statistical differences were determined by the two-tailed $t$ test between two groups. $* P<0.05 ; * * * P<$ 0.005 compared with MCD group. during the last 2 weeks of MCD feeding. To test the preventive and therapeutic effect of WA in the HFD-induced NASH model, mice were fed an HFD or LFD for 20 weeks. Mice were fed an HFD or LFD for 8 weeks and then divided into four groups: 1) mice treated with vehicle for 12 weeks; 2) mice treated with WA for 12 consecutive weeks; 3 ) mice treated with vehicle for 4 weeks from week 9 to week 12 , and then treated with WA for the last 8 weeks; and 4) mice treated with vehicle for 8 weeks from week 9 to week 16, and then treated with WA for 4 weeks. To test the therapeutic effect of WA in leptin-deficient NASH, the leptin-deficient ob/ob NASH mouse model was used. Eight-weekold age-matched ob/ob mice, obtained from Jackson Laboratories, were fed an HFD for 8 weeks and then divided into two groups: 1) mice maintained on an HFD and treated with WA or control vehicle for 4 weeks, and 2) mice maintained on an HFD and treated with WA for 4 weeks. The mice were injected with vehicle or WA before the dark cycle of the day and not fasted during the study. One hour after the last injection of WA or control vehicle, the mice were killed with $\mathrm{CO}_{2}$, and serum and tissues were collected for further analysis.

Histology Analysis. Formalin-fixed liver tissues were embedded in paraffin. A portion of fresh livers were embedded in Tissue-Tek Optimal Cutting Temperature compound (Sakura Finetek, Torrance, CA) and then flash frozen for Oil Red O (VitroVivo, Biotech, Rockville, MD) staining. Five-micrometer-thick sections were cut for both H\&E and picrosirius red staining. Further sample processing, analysis for haematoxylin and eosin (H\&E) staining, and Oil Red O staining were performed at Histoserv Inc. (Germantown, MD) or VitroVivo Biotech. For picrosirius red staining in Fig. 8, paraffin-embedded livers were sectioned at Histoserv Inc. and stained with picrosirius, whereas the picrosirius staining in Fig. 12 was performed at VitroVivo Biotech. Digital images from ppicrosirius red-stained liver sections were analyzed with Image-Pro Plus software (Media Cybernetics, Inc., Rockville, MD), and the collagen-positive area was assessed. Large blood vessels and corner sections were excluded from analysis, and at least five different fields of each slide were measured to allow a realistic picture of the entire organ's fibrotic state. Results were expressed as collagen percentage of all images related to the total area of the tissue.

Biochemical Analysis of Liver and Serum. Biochemical parameters including levels of ALT, AST, TC, TG, and NEFAs, as well as levels of superoxide dismutase, hydroxide peroxide, and glutathione in serum or liver were tested with commercial kits based on the manuals. Serum ceramides were quantified as described previously (Xie et al., 2017).

Quantitative Polymerase Chain Reaction. The livers were flash frozen in liquid nitrogen and stored at $-80^{\circ} \mathrm{C}$. Total RNA from frozen livers was extracted with TRIzol reagent (Invitrogen, Carlsbad, CA). cDNA was synthesized from $1 \mu \mathrm{g}$ of total RNA using qScript cDNA SuperMix (Quantabio, Beverly, MA). Analysis was performed by using the ABI PRISM 7900 Sequence Detection System (Applied Biosystems, Bedford, MA). Values were normalized to Actb or glyceraldehyde3-phosphate dehydrogenase mRNAs and the results expressed as fold change relative to the control group. Primer sequences are listed in Supplemental Table 1.

Pharmacokinetic Analysis. Age-matched and body weight-matched 8-week-old C57BL/6N mice were randomly grouped and dosed with $5 \mathrm{mg} / \mathrm{kg}$ WA via intraperitoneal administration. Blood was collected at 0 minutes (predose), 10 minutes, 20 minutes, 40 minutes, 1 hour, 2 hours, 4 hours, 8 hours, 24 hours, and 48 hours for isolation of plasma. Samples were diluted with acetonitrile, and protein was removed by centrifugation and then analyzed using ultraperformance liquid chromatography-tandem mass spectrometry under reverse-phase chromatography after optimizing chromatographic conditions for WA. MassLynx software version 4.1 (Waters Corp, Milford MA) was used to analyze the drug concentration data. The pharmacokinetic parameters of WA were calculated by the noncompartmental model using WinNonlin software version 5.2.1 (Pharsight Corporation, Sunnyvale, $\mathrm{CA}$ ). The $\mathrm{C}_{\max }$ values and the time to reach maximum plasma concentration $\left(\mathrm{T}_{\max }\right)$ were calculated directly from the observed plasma concentration versus time data. The area under the plasma drug concentration-time curve from time 0 to 48 hours $\left(\mathrm{AUC}_{0-48} \mathrm{~h}\right)$ was calculated using the linear trapezoidal rule. The $\mathrm{AUC}_{0-\text { infinity }}$ was calculated as follows: $\mathrm{AUC}_{0-\text { infinity }}=\mathrm{AUC}_{0-48 \mathrm{~h}}+\mathrm{C}_{\mathrm{t}} / \mathrm{K}_{\mathrm{el}}$, where $\mathrm{C}_{\mathrm{t}}$ is the last plasma concentration measured, and $\mathrm{K}_{\mathrm{el}}$ is the elimination rate constant; $\mathrm{K}_{\mathrm{el}}$ was determined using linear regression analysis of the logarithm linear part of the plasma concentration-time curve. The $t_{1 / 2}$ of WA was calculated as $\mathrm{t}_{1 / 2}=\ln 2 / \mathrm{K}_{\mathrm{el}}$. 

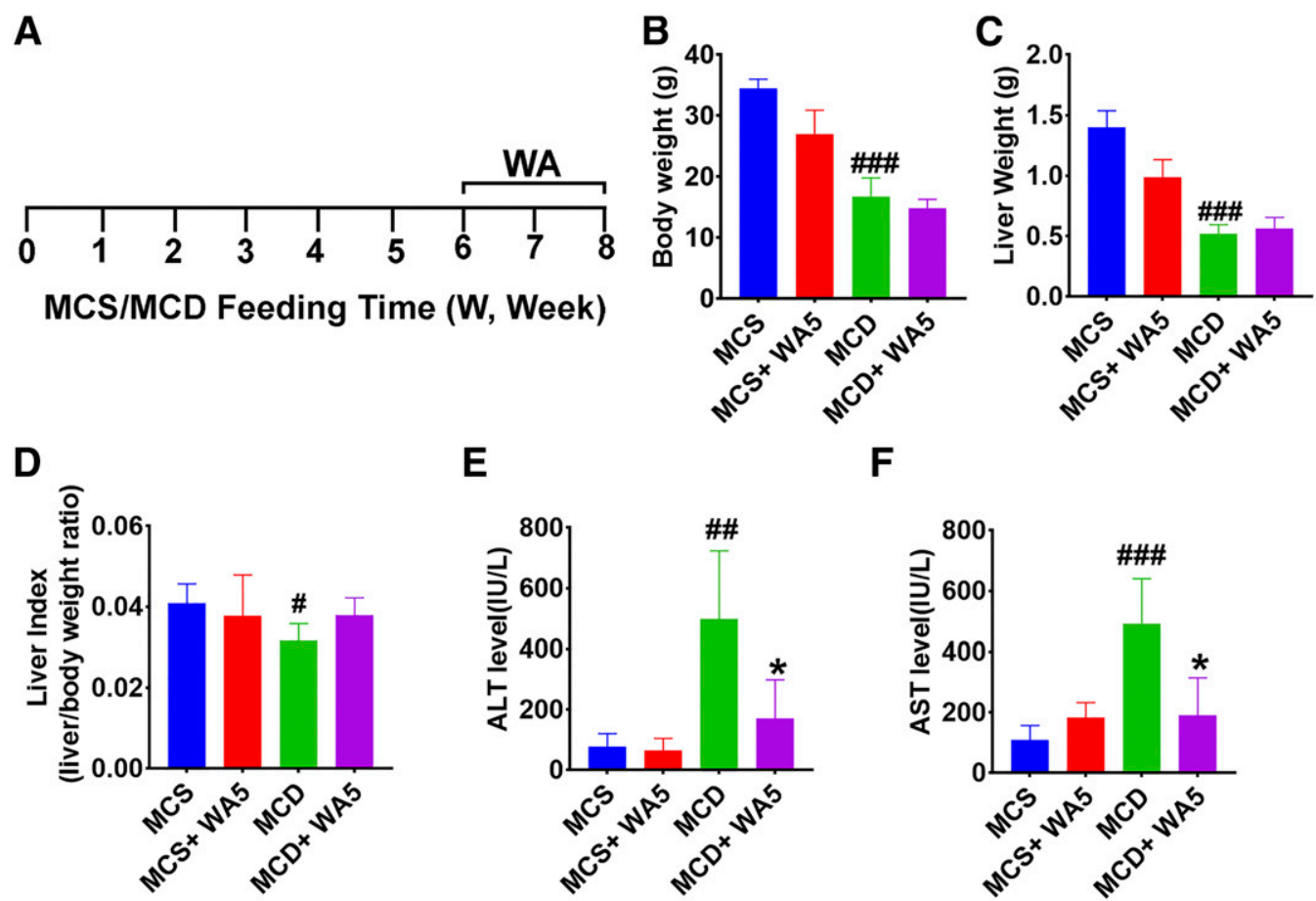

E

$\mathbf{F}$

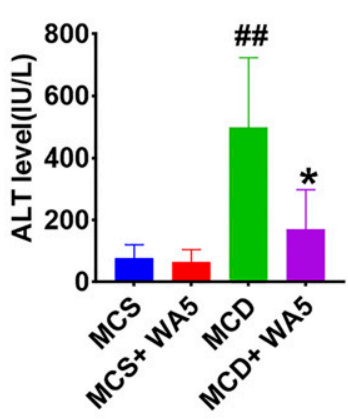

H

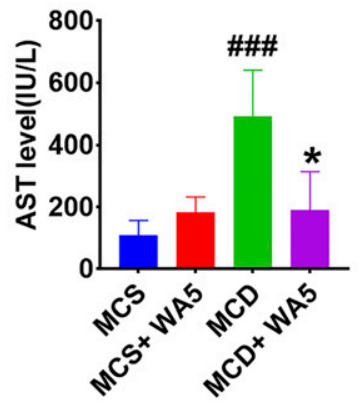

$H \& E$
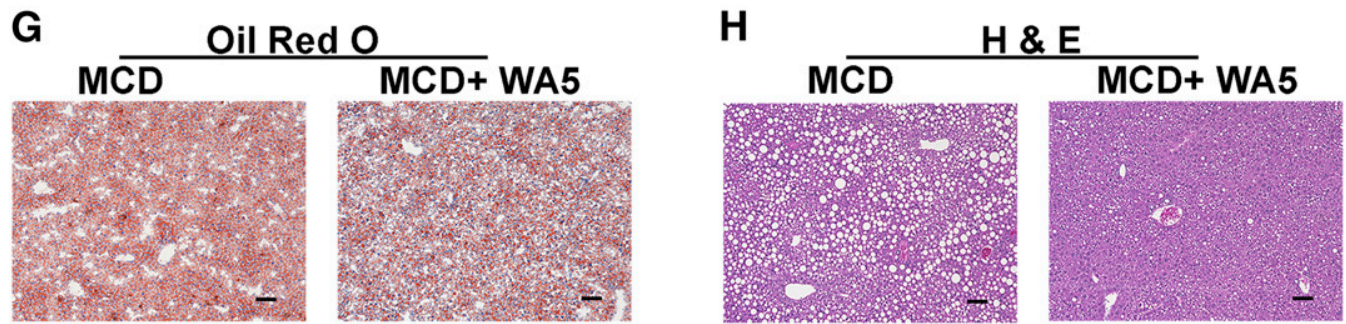

Fig. 4. WA therapeutically improved MCD diet-induced NASH. (A) Experiment scheme for testing the therapeutic effect of WA in the MCD-induced NASH model. (B) Effect of $5 \mathrm{mg} / \mathrm{kg}$ WA on body weight. (C) Effect of $5 \mathrm{mg} / \mathrm{kg}$ WA on liver weight. (D) Effect of $5 \mathrm{mg} / \mathrm{kg}$ WA on liver indexes. (E and F) Analysis of serum ALT (E) and AST (F) levels in LFD-fed mice and HFD-fed mice treated with control vehicle or WA. (G and H) Histology analysis of hepatic steatosis by Oil Red O staining $(G)$ and H\&E staining $(H)$. Scale bar, $100 \mu \mathrm{m}$. Data were presented as means \pm S.D. Descriptions for the MCS, MCS+WA5, MCD, and MCD+WA5 groups were the same as described in the legend to Fig. $1 . n=5$ mice per group. ${ }^{\#} P<0.05$; ${ }^{\# \#} P<0.01 ;{ }^{\# \# \#} P<0.005$ when compared with the MCS group; $* P<0.05$ when compared with the MCD group. Statistical differences were determined by the two-tailed $t$ test or one-way ANOVA.

Statistical Analysis. Experimental values were presented as the mean \pm S.D. Statistical analyses were determined by two-tail $t$ test between two groups or by one-way ANOVA followed by Dunnett's multiple comparisons test among multiple groups using Prism version 7.0 (GraphPad Software, San Diego, CA). $P$ values less than 0.05 were considered statistically significant.

\section{Results}

WA Dose-Dependently Attenuates MCD Diet-Induced Liver Injury. To explore the effective dose of WA in the MCD diet-induced liver injury model, WA at $1,2.5$, and $5 \mathrm{mg} / \mathrm{kg}$ was administered. From the first day of MCD diet feeding, WA was injected once a day for four consecutive weeks (Fig. 1A). The MCD diet induced a significant loss of body weight, which was significantly attenuated by $5 \mathrm{mg} / \mathrm{kg}$ WA (Fig. 1B). WA dosedependently increased the liver weight and liver index in mice on the MCD diet (Fig. 1, C and D). WA treatment alone caused no significant histologic changes suggestive of liver damage (Fig. 1E) and did not significantly affect serum ALT and AST levels (Fig. 2, A and B) in the control MCS diet group, indicating that WA at $5 \mathrm{mg} / \mathrm{kg}$ was not hepatotoxic. Further biochemical assays showed that WA dose-dependently inhibited the MCD diet-induced increase of serum ALT and AST levels (Fig. 2, A and B) as well as hepatic levels of TC and TG (Fig. 2, $\mathrm{C}$ and $\mathrm{D})$. The MCD diet induced a marked decrease of serum $\mathrm{TC}$ and TG, which was significantly attenuated by $5 \mathrm{mg} / \mathrm{kg}$ WA (Fig. 2, E and F). Given that WA at $5 \mathrm{mg} / \mathrm{kg}$, among the tested doses, showed a consistent effect in reversing body weight changes, serum ALT and AST levels, and TC and TG levels in livers and serum, the effect of WA on hepatic inflammation and histology was measured at this dose. WA alleviated MCD diet-induced liver inflammation (Fig. 2G), and histologic analyses by $\mathrm{H} \& \mathrm{E}$ and Oil Red $\mathrm{O}$ staining showed that $5 \mathrm{mg} / \mathrm{kg}$ WA improved MCD diet-induced hepatic steatosis (Fig. 2, $\mathrm{H}$ and I). These data demonstrate that WA dosedependently prevents MCD diet-induced fatty liver, and that the $5-\mathrm{mg} / \mathrm{kg}$ dose of WA ameliorates NASH.

With a dose of $5 \mathrm{mg} / \mathrm{kg}$ WA chosen for further study, a pharmacokinetic analysis was performed to investigate the exposure and clearance behavior of WA in blood after intraperitoneal injection. The chemical structure of WA and drug 


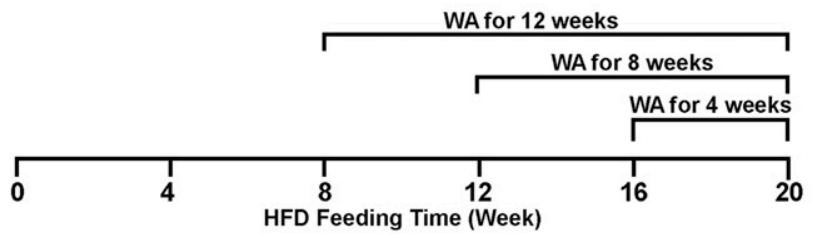

B
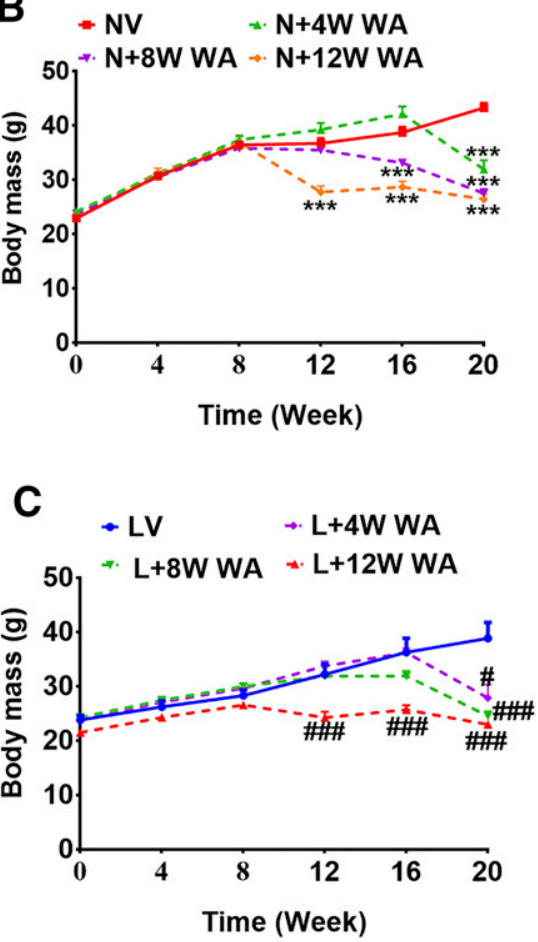

D

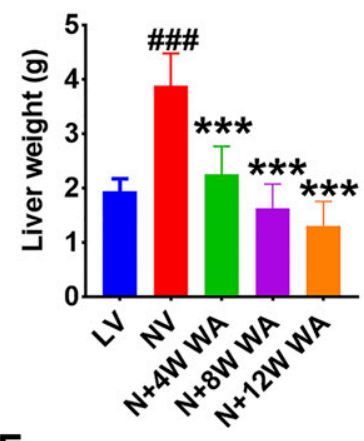

$\mathbf{F}$

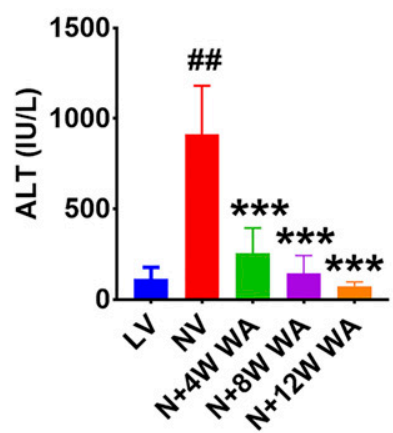

E

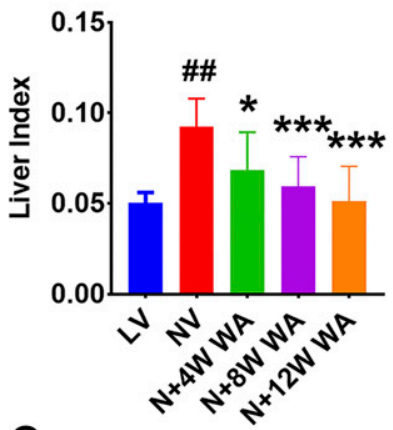

G

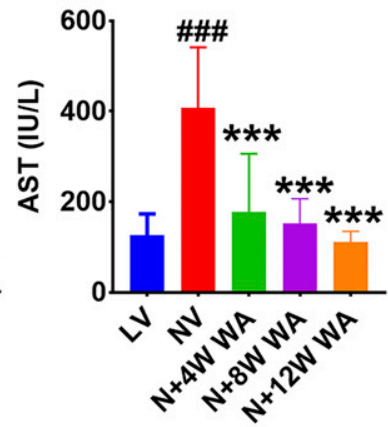

Fig. 5. WA improved HFD-induced liver injury. (A) Experiment scheme of the time-course dosing regimen. (B and C) Body weights of HFD-fed mice (B) and LFD-fed mice (C) treated with vehicle or WA. (D and E) Liver weights (D) and liver indexes calculated by liver weight/body weight ratio (E). (F and G) Analysis of serum ALT (F) and AST (G) levels. Data were presented as means \pm S.D. LV, mice fed the LFD and treated with vehicle (V); L $+4 \mathrm{~W}$ WA, mice fed LFD and treated with WA for the last 4 weeks; L +8 W WA, mice fed the LFD and treated with WA for the last 8 weeks; L +12 W WA, mice fed the LFD and treated with WA for the last 12 weeks; NV, mice fed the HFD-induced NASH diet (N) and treated with vehicle; N $+4 \mathrm{~W}$ WA, mice fed the HFDinduced NASH diet and treated with WA for the last 4 weeks; N+8W WA, mice fed the HFD-induced NASH diet and treated with WA for the last 8 weeks; $\mathrm{N}+12 \mathrm{~W}$ WA, mice fed the HFD-induced NASH diet and treated with WA for the last 12 weeks. $n=5$ mice for the LFD-fed groups and $n=7-10$ for HFD-fed mice. ${ }^{\# \#} P<0.01$; ${ }^{\# \# \#} P<0.005$ compared with the LFD group; ${ }^{*} P<0.05$; ${ }^{* * *} P<0.005$ compared with the HFD group. Statistical differences were determined by the two-tailed $t$ test or one-way ANOVA.

concentration-time curve of WA are shown (Supplemental Fig. 1, A and B). The pharmacokinetic parameters, including $\mathrm{C}_{\text {max }}, \mathrm{t}_{1 / 2}, \mathrm{AUC}_{0-48 \mathrm{~h}}, \mathrm{AUC}_{0-\infty}$, and elimination rates $\left(\mathrm{K}_{\mathrm{eli}}, 1 / \mathrm{h}\right)$, were calculated (Supplemental Fig. 1C). Following a single intraperitoneal injection dose of $5 \mathrm{mg} / \mathrm{kg} \mathrm{WA}$, a $\mathrm{C}_{\max }$ level of $14.3 \pm 1.8 \mathrm{nM}$ was observed at 20 minutes. The plasma $t_{1 / 2}$ value of WA was $2.0 \pm 0.6$ hours. These data and a previous study (Thaiparambil et al., 2011) that found intraperitoneally injected WA had a half-life of 1.36 hours, suggest that WA is rapidly clearance from blood after intraperitoneal dosing. However, WA was readily detected across the time course of 48 hours and was maintained at $2-4 \mathrm{nM}(14 \%-29 \%$ of WA peak concentrations) at the later phase after a single dose of $5 \mathrm{mg} / \mathrm{kg}$ WA (Supplemental Fig. 1B). These data suggest that WA could be maintained at a relatively low level for 48 hours while having a short half-life of 2 hours in mice.

WA Prevents MCD Diet-Induced Liver Injury Independent of Body Weight Change. To examine whether WA could prevent MCD diet-induced NASH independent of its effect in changing MCD diet-induced body weight loss, a short-term MCD diet-feeding study was performed. Mice were fed an MCD diet for 10 days, during which $5 \mathrm{mg} / \mathrm{kg}$ WA or control vehicle was injected once a day for 10 consecutive days (Fig. 3A). In this short-term study, $5 \mathrm{mg} / \mathrm{kg}$ WA did not change the body mass of MCD diet-fed mice (Fig. 3B). However, WA markedly increased the liver weight and the liver index when compared with control vehicle-treated controls (Fig. 3, C and D). WA significantly decreased the MCD diet-induced serum ALT and AST levels (Fig. 3, E and F). These data demonstrate that $5 \mathrm{mg} / \mathrm{kg}$ WA is sufficient to alleviate MCD diet-induced liver injury at the early stages of NASH in the absence of significant body weight changes.

WA Therapeutically Improves MCD Diet-Induced Liver Injury. To further explore whether WA could therapeutically treat MCD diet-induced NASH, $5 \mathrm{mg} / \mathrm{kg}$ WA was administered for 2 weeks after the onset of liver damage induced by 6 weeks of MCD diet feeding (Fig. 4A). WA did not affect the body weight, liver weight, or liver index (Fig. 4, B-D), whereas it significantly decreased the MCD diet-induced increases of serum ALT and AST levels (Fig. 4, E and F). 
A

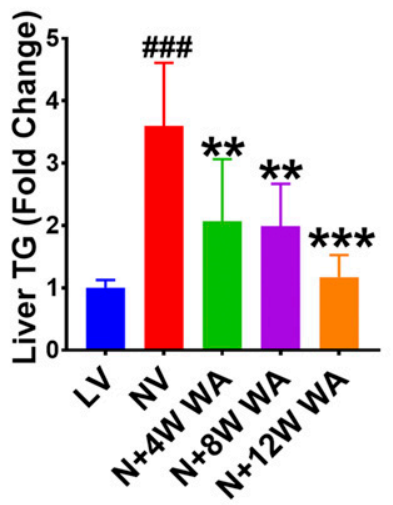

D

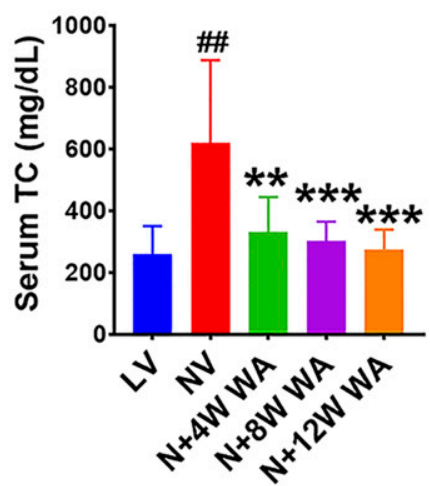

B



E

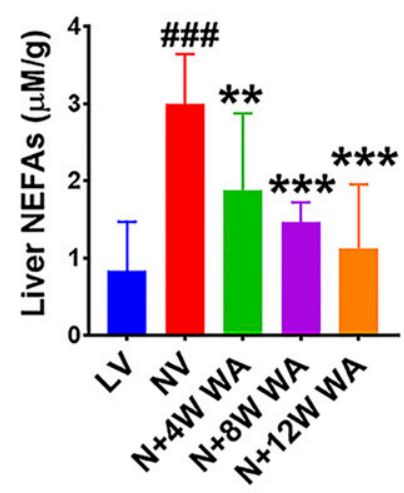

C

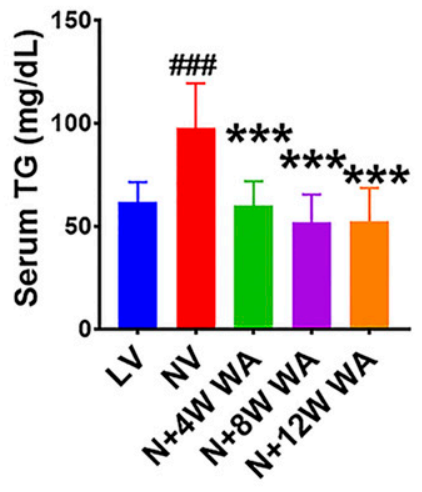

$\mathbf{F}$

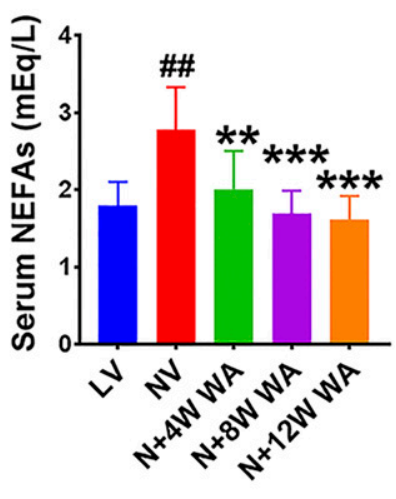

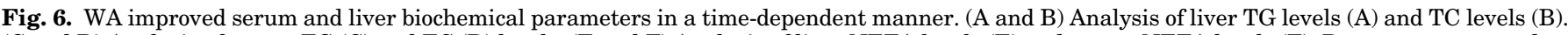

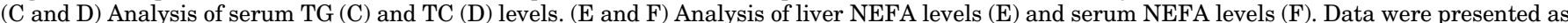

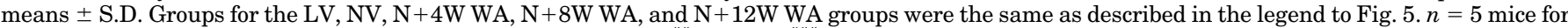

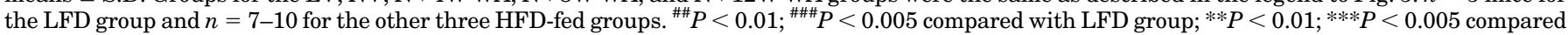
with the HFD group. Statistical differences were determined by the two-tailed $t$ test or one-way ANOVA.

WA also improved hepatic steatosis as revealed by histologic analyses (Fig. 4, G and H). These data suggest that $5 \mathrm{mg} / \mathrm{kg}$ WA therapeutically improves MCD diet-induced liver injury.

WA Improves HFD-Induced Liver Injury. In contrast to the MCD diet-induced NASH model that results in body weight loss and does not cause insulin resistance coincident with inducing steatohepatitis, the $40 \mathrm{kcal} \%$ HFD-induced obese NASH model is thought to better mimic the typical human NASH pathologies of insulin resistance, high serum lipid accumulation, and obesity (Hebbard and George, 2011; Griffett et al., 2015; Honda et al., 2016; Ding et al., 2018). To further confirm the effect of WA in treating NASH, the HFD regimen and its matched LFD were used to test the effect of WA both in preventing and therapeutically improving NASH. Mice were fed an HFD for 20 weeks and injected with $5 \mathrm{mg} / \mathrm{kg}$ WA or control vehicle for the last 12,8 , or 4 weeks before killing (Fig. 5A). WA significantly decreased body weights in both the HFD-fed and LFD-fed mice (Fig. 5, B and C). WA also decreased liver weights and liver indexes (Fig. 5, D and E) and attenuated the HFD-induced increase of serum ALT and AST (Fig. 5, F and G) in a time-dependent manner. Similarly, WA treatment decreased the HFD-induced accumulation of hepatic TG and TC levels (Fig. 6, A and B), and serum TG and TC levels (Fig. 6, C and D). WA treatment time-dependently decreased the HFD-induced accumulation of NEFAs in both livers and serum (Fig. 6, E and F). Further histologic analyses showed that WA treatment time-dependently improved NASHassociated hepatic steatosis as revealed by histologic analyses (Fig. 7, A and B). WA treatment also significantly reduced the levels of the HFD-induced Tnfa, interleukin $1 \mathrm{~b}$ (Il1b), and Il6 mRNAs involved in hepatic inflammation (Fig. 7, C-E), and heat shock protein family A member 5 (Hspa5) and DNA damage-inducible transcript 3 (Ddit3) mRNA levels involved in ER stress (Fig. 7, F and G). WA treatment decreased the HFD-induced increase of sterol regulatory element-binding protein 1c (Srebp1c) mRNA encoding the transcription factor SREBP1C and its downstream target gene mRNAs, Scd1 and Fas, involved in the control of lipogenesis (Fig. 7, H-J). These data demonstrate that WA treatments both prevent and therapeutically improve HFD-induced hepatic steatosis, inflammation, and ER stress accompanied by dampening of the hepatic lipogenesis signaling.

WA Attenuates Hepatic Fibrosis. Fibrosis is a major hallmark of NAFLD progression to NASH. Twenty-week HFD feeding markedly induced picrosirius red staining in the NASH group, which was rescued by WA treatment in a time-dependent manner (Fig. 8A). Further statistical analysis showed that WA treatment decreased NASH-associated hepatic collagen accumulation (Fig. 8B). In addition, WA treatment decreased Timp2, Col1a1, Mmp2, and Acta2 mRNAs encoding enzymes and structural proteins involved in liver fibrogenesis (Fig. 8, C-F). These data demonstrate that WA 
A

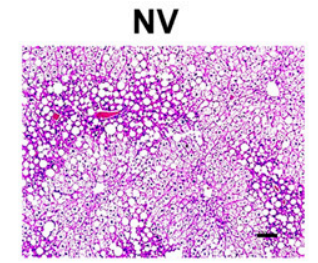

B

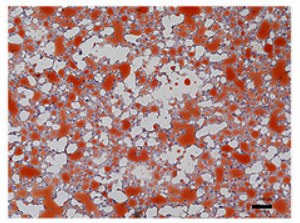

$N V+4 W$ WA
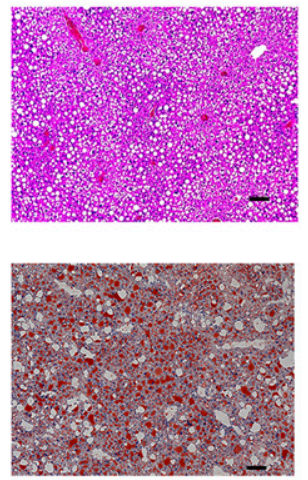

$N V+8 W$ WA


$N V+12 W$ WA
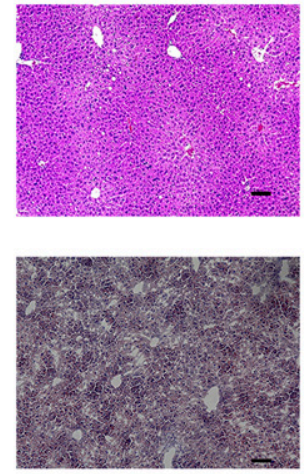

$\mathbf{F}$

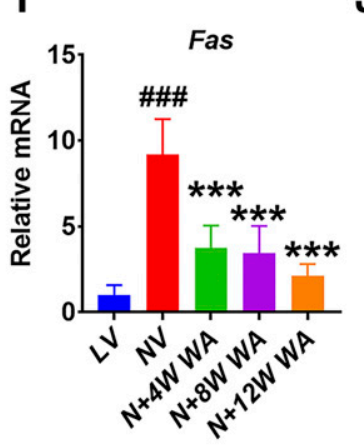

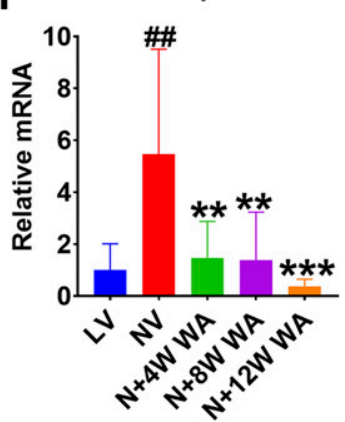

J


E

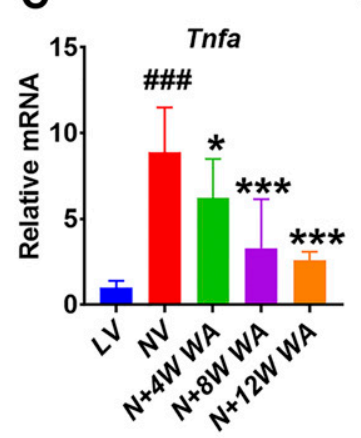

G

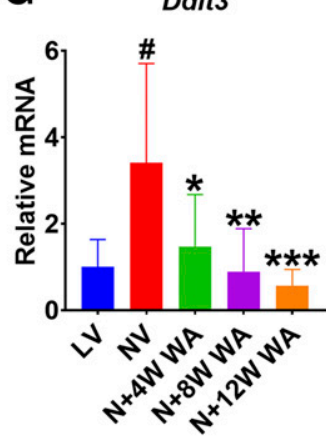

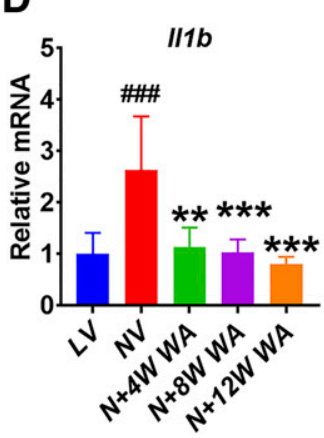

H



Fig. 7. WA improved HFD-induced hepatic steatosis and inflammation. (A and B) Representative histology pictures of H\&E staining (A) and Oil Red O staining (B). Scale bar, $100 \mu \mathrm{m}$. (C-E) Effect of WA on HFD-induced liver Tnfa, Il1b, and $I l 6$ mRNA levels. (F and G) Effect of WA on HFD-induced hepatic ER stress signaling marker (Hspa5 and Didt3) mRNA levels. (H-J) Effect of WA on HFD-induced Srebp 1c, Fas, and Scd1 mRNA levels. Data were presented as means \pm S.D. Descriptions for the LV, NV, N $+4 \mathrm{~W}$ WA, N $+8 \mathrm{~W}$ WA, and $\mathrm{N}+12 \mathrm{~W}$ WA were the same as described in the legend to Fig. 5. $n=5$ mice for the LFD group and $n=7-10$ for the other three HFD-fed groups. ${ }^{\# \# \# ~} P<0.005$ compared with LFD group; ${ }^{*} P<0.05$; ${ }^{* *} P<0.01$; $* * * P<0.005$ compared with the HFD group. Statistical differences were determined by the two-tailed $t$ test or one-way ANOVA.

improves NASH-associated fibrosis induced by HFD in a timedependent manner.

WA Rescues NASH-Associated Ceramide Accumulation, ER Stress, and Oxidative Stress. Previous studies demonstrated that HFD-induced hepatic steatosis was correlated with increased ceramide levels (Longato et al., 2012; Chaurasia and Summers, 2015; Kasumov et al., 2015). Genetically or biochemically decreasing ceramides improved NAFLD and NASH (Kurek et al., 2014; Xie et al., 2017). Oxidative stress that occurs in NASH could induce ceramide accumulation (Bikman and Summers, 2011), and WA has exhibited antioxidant activity in earlier experimental models (Vanden Berghe et al., 2012). Thus, the antioxidant effects of WA could potentially influence ceramide levels and oxidative stress when treating NASH. To examine this hypothesis, levels of serum ceramides were first measured using authentic standards. HFD significantly induced the accumulation of ceramides, such as C16 (m/z 582.5098), $\mathrm{C} 18$ (m/z 610.5411), C20 (m/z 638.5724), C22 (m/z 666.6037), $\mathrm{C} 24(\mathrm{~m} / \mathrm{z} 694.6350), \mathrm{C} 18: 1$ (m/z 608.5254), and C24:1 (m/z 692.6193), all of which were reduced by WA treatment (Fig. 9, A-G).

Next, the possibility that WA attenuated HFD-induced oxidative stress was examined. WA treatment time-dependently rescued the NASH-induced decrease of hepatic superoxide dismutase and glutathione levels, and significantly attenuated the NASH-induced increase of hepatic peroxide levels (Fig. 9, H-J). These data suggest that WA treatment reduces HFD-induced ceramide accumulation in serum and oxidative stress in liver. 
A

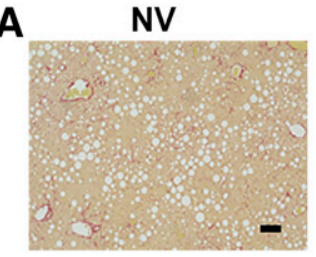

LV

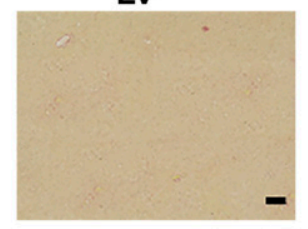

B

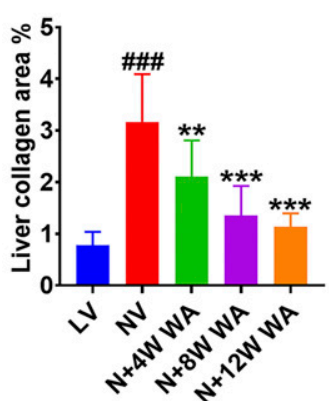

$\mathbf{F}$

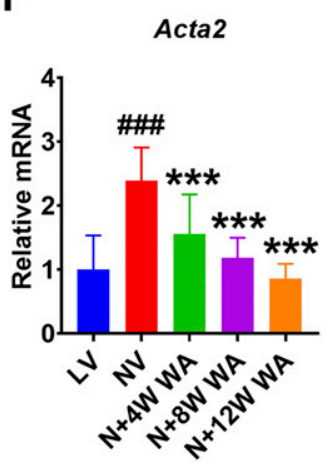

$\mathrm{N}+$ 4W WA

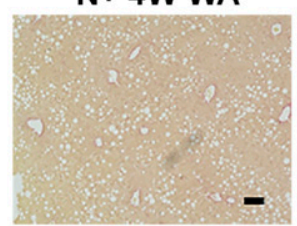

L+4W WA

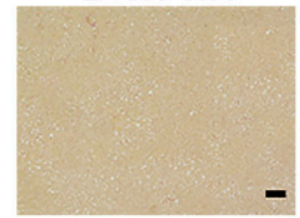

C

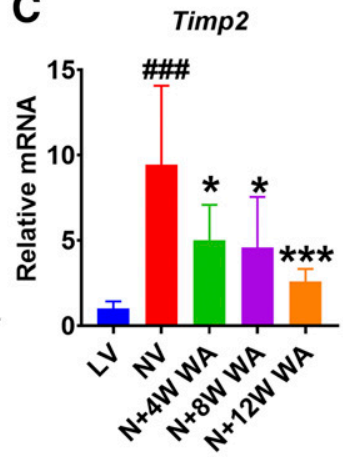

D
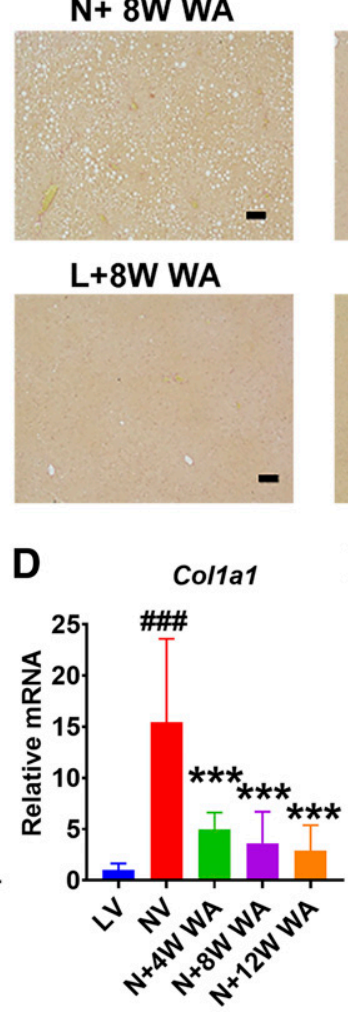

L+8W WA

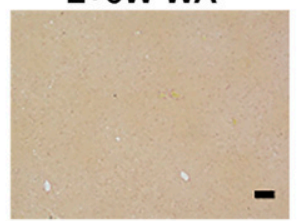

$\mathrm{N}+12 \mathrm{~W}$ WA

E

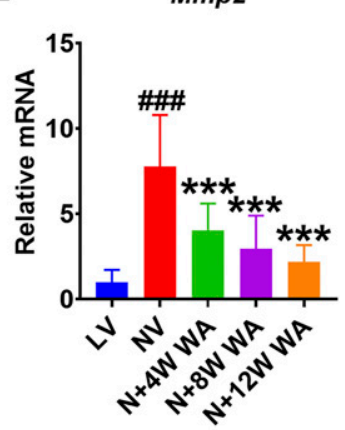

To further examine why WA alleviated serum ceramide accumulation and hepatic oxidative stress, mRNA levels of ceramide signaling and NRF2 signaling were analyzed. WA treatment markedly decreased mRNAs of genes involved in ceramide biosynthesis pathways, such as serine palmitoyltransferase, long-chain base subunit 1 (Sptlc1) and Sptlc2; sphingomyelin phosphodiesterase 1 (Smpd1), Smpd2, Smpd3, and Smpd4; alkaline ceramidase 2 (Acer2) and Acer3; sphingomyelin synthase (Sgms1) and Sgms2; and ceramide synthase 2 (Cers2), Cers4, and Cers6, in a time-dependent manner (Fig. 10). These data demonstrate that WA attenuates HFD-induced accumulation of serum ceramides and oxidative stress. Further analysis of NRF2 signaling revealed that WA decreased mRNA levels of genes involved in the NRF2 pathway in both 20-week HFD-treated mice (Supplemental Fig. 2) and 4-week MCD diet-treated mice (Supplemental Fig. 3A). Given that earlier reports revealed that WA could activate NRF2 (Jadeja et al., 2015; Heyninck et al., 2016; Palliyaguru et al., 2016), the possibility exists that the decreases in NASH diet-induced NRF2 signaling by WA in the current study are due to the secondary results of its hepatoprotective effects. The marked hepatoprotective effect may decrease NASH diet-promoted NRF2 signaling, which could overcome the effect of WA on NRF2 activation under the pathologic conditions of NASH. To answer this question, the effect of WA on the hepatic NRF2 signaling pathway at an early stage of the NASH model induced by the MCD diet or HFD was examined. Mice were fed an MCD diet for 10 days or an HFD for 1 week, during which mice were cotreated with control vehicle or $5 \mathrm{~m} / \mathrm{kg}$ WA once a day via intraperitoneal injection, and all mice were killed 1 hour after the last WA injection to collect livers for further mRNAs analysis. In 10-day MCD diet-fed mice, WA was found 

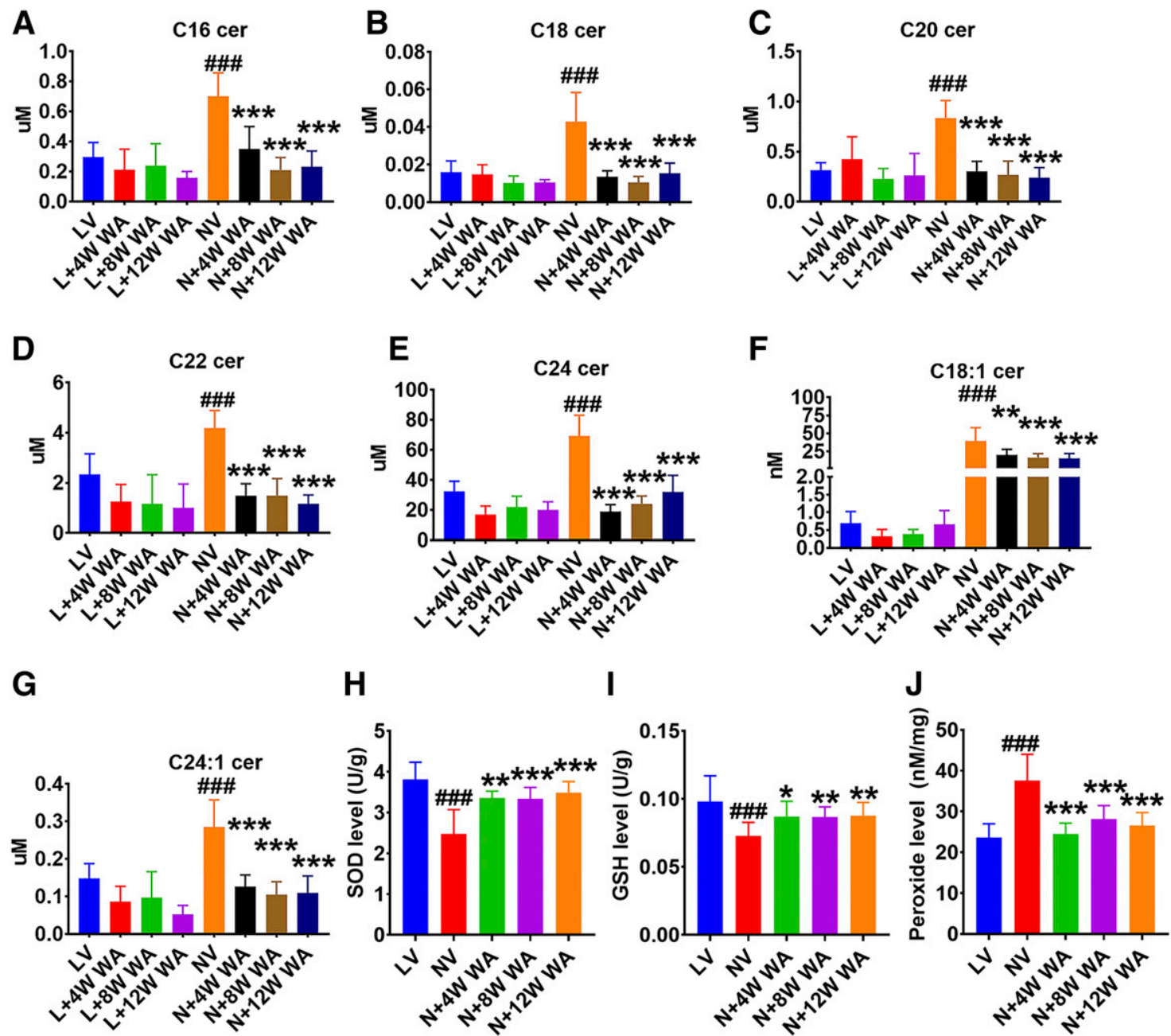

Fig. 9. WA rescued HFD-induced ceramide accumulation and oxidative stress. (A-G) Quantitation of serum ceramide concentrations for C16 (A), C18 (B), C20 (C), C22 (D), C24 (E), C18:1 (F), and C24:1 (G). (H-J) Analysis of hepatic superoxide dismutase (SOD) levels (H), glutathione (GSH) levels (I), and peroxide levels $(J)$. Data were presented as means \pm S.D. Descriptions for the LV, NV, N $+4 \mathrm{~W}$ WA, N $+8 \mathrm{~W}$ WA, and N+12W WA groups were the same as described in the legend to Fig. 5. $n=5$ mice for the LFD-fed groups and $n=7-10$ for the HFD-fed groups. ${ }^{\# \# \#} P<0.005$ compared with the LFD group; $* P<0.05$; ** $P<0.01$; *** $P<0.005$ compared with HFD group. Statistical differences were determined by the two-tailed $t$ test or one-way ANOVA.

to induce hepatic mRNAs of NRF2 target genes, including Gclc, Nqo1, Keap1, Cat, and Nrf2 (Supplemental Fig. 3B), whereas in 1-week HFD-treated mice, this NRF2-activating effect of WA was not found. These data suggest that WA may affect the mRNA expression of NRF2 target genes in a contextdependent manner.

WA Improves NASH in HFD-Induced Liver Injury in Leptin Signaling-Defective (ob/ob) Mice. To determine the effect of WA in a leptin-deficient NASH model, ob/ob mice were fed an HFD for 12 weeks and injected with $5 \mathrm{mg} / \mathrm{kg}$ WA or control vehicle for the last 4 weeks before killing (Fig. 11A). WA significantly decreased body weight, liver weight, and liver index (Fig. 11, B-D), and attenuated the HFD-induced increase of serum ALT and AST (Fig. 11, E and F). Histologic analyses revealed that WA treatment improved NASH-associated hepatic steatosis (Fig. 11, G and H). Consistent with this finding, WA treatment reduced the mRNA levels of proinflammatory cytokines, including $I l 1 b, I l 6$, and Tnfa mRNAs, as well as the mRNA levels of lipogenesis genes, including Srebp1c, Scd1, and Fas (Fig. 11I). WA treatment decreased the HFD-induced accumulation of TG, TC, and NEFAs in liver and serum (Fig. 12, A-F). Furthermore, WA also significantly reduced the
mRNA levels of the fibrogenesis genes Timp2, Col1a1, Mmp2, and Acta2 (Fig. 12G) and attenuated HFD-induced hepatic fibrosis as revealed by picrosirius red staining data (Fig. 12H). Similar to its effect in HFD-treated C57BL/6N mice, WA also significantly decreased HFD-induced ER stress marker Hspa5 and Ddit3 mRNAs, as well as all of the tested mRNAs involved in ceramide synthesis, including $S p t l c 1, S p t l c 2, S m p d 2, S m p d 3$, Acer2, Sgms1, Sgms2, Cers2, Cers4, and Cers6 (Fig. 12, I and J). These results demonstrate that WA treatment decreases HFD-induced NASH in ob/ob mice, and this was accompanied by decreased hepatic ceramide synthesis.

\section{Discussion}

The hepatoprotective roles of WA in treating liver diseases, especially the effects on NASH, are largely unknown. The current study revealed that WA could both prevent and therapeutically improve NASH in two widely-used and well-defined mouse NASH models, the MCD-induced NASH model and the HFD-induced NASH model in C57BL/6N mice, and could therapeutically alleviate an ob/ob mouse leptin-deficient HFD-induced NASH modeli. WA restored NASH-induced 
A

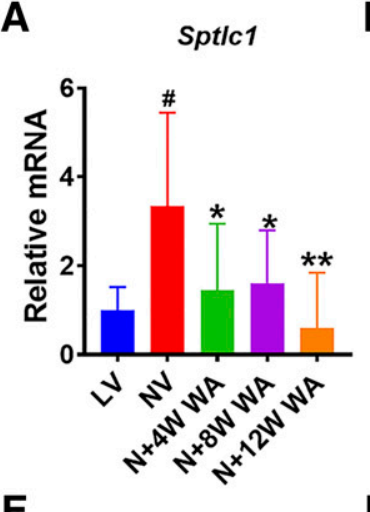

E

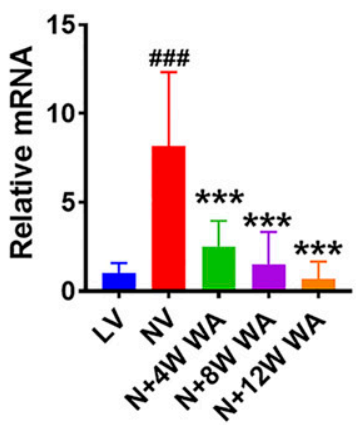

I

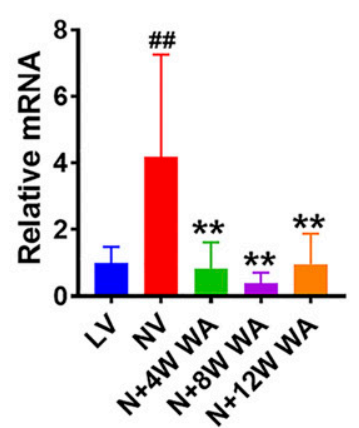

M

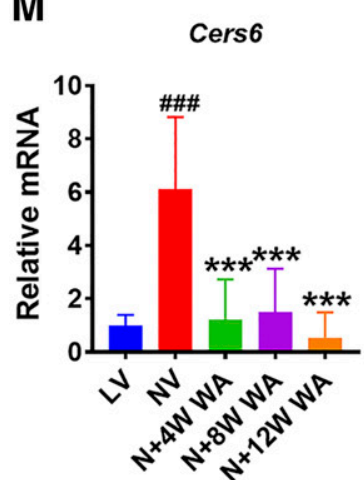

B

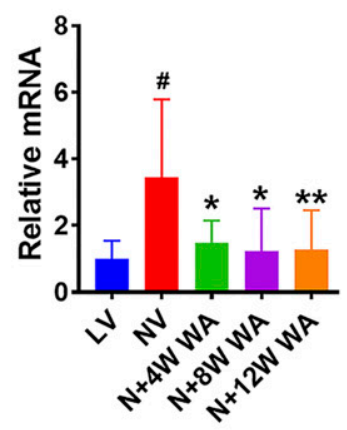

F

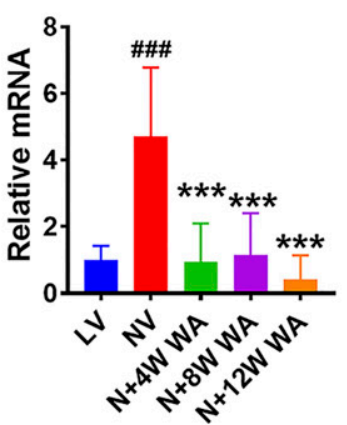

Sgms2

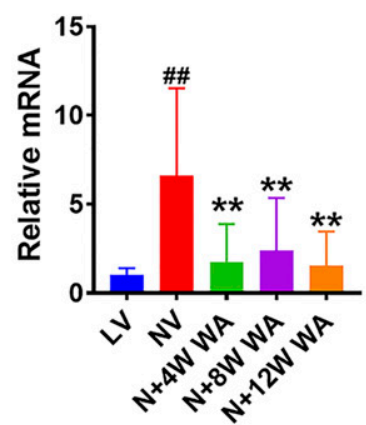

C

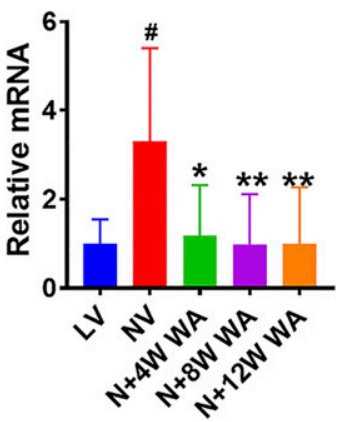

G
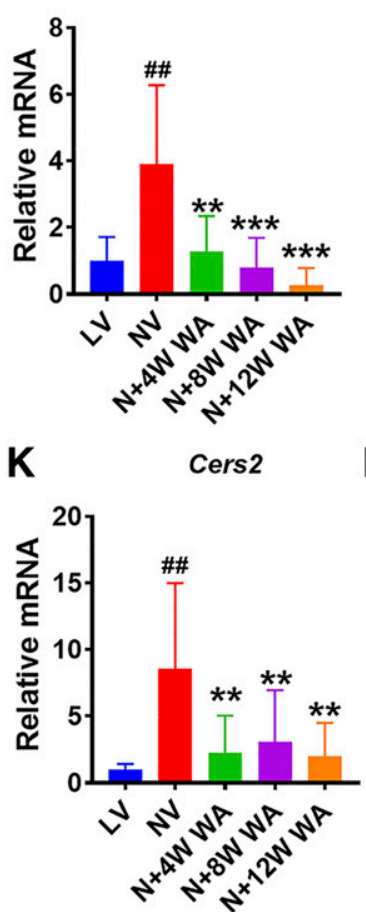

D $\quad$ Smpd2

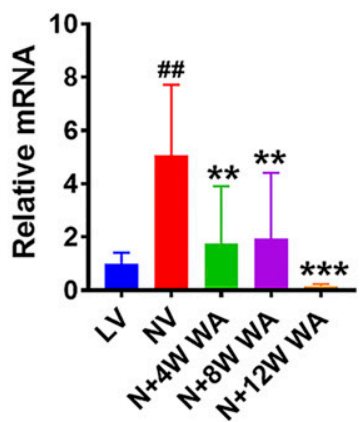

H

Acer3

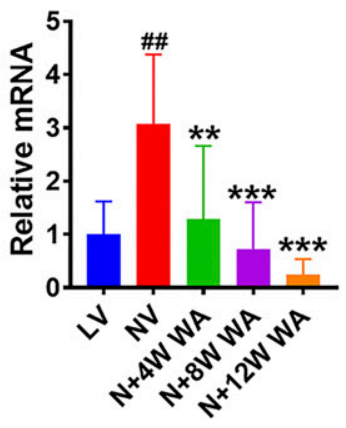

L Cers4

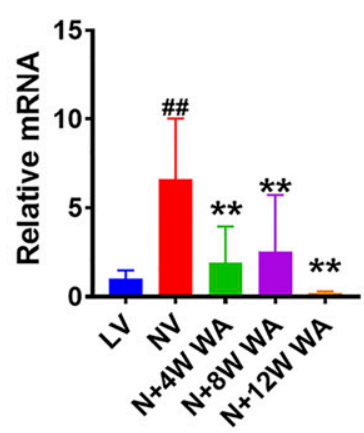

Fig. 10. WA treatment reduced ceramide biosynthesis and catabolism in WA-treated HFD-fed C57BL/6N mice and ER stress markers in a timedependent manner. Ceramide biosynthesis and catabolism marker analysis of Sptlc1 (A), Sptlc2 (B), Smpd1 (C), Smpd2 (D), Smpd3 (E), Smpd4 (F), Acer2 (G), Acer3 (H), Sgms1 (I), Sgms2 (J), Cers2 (K), Cers4 (L), and Cers6 (M) mRNA levels. Descriptions for the LV, NV, N+4W WA, N+8W WA, and $\mathrm{N}+12 \mathrm{~W}$ WA were the same as described in the legend to Fig. 5. $n=5$ mice for the LFD group and $n=7-10$ for the other three HFD-fed groups. ${ }^{\# \# \#} P<$ 0.005 compared with the LFD group; $* P<0.05 ; * * P<0.01 ; * * * P<0.005$ compared with the HFD group. Statistical differences were determined by the two-tailed $t$ test or one-way ANOVA. 
A

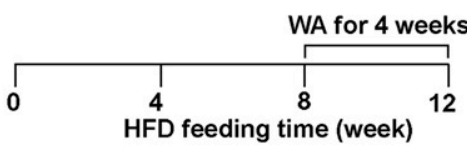

B


H NV

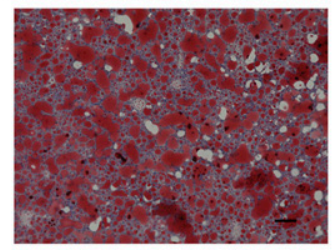

C
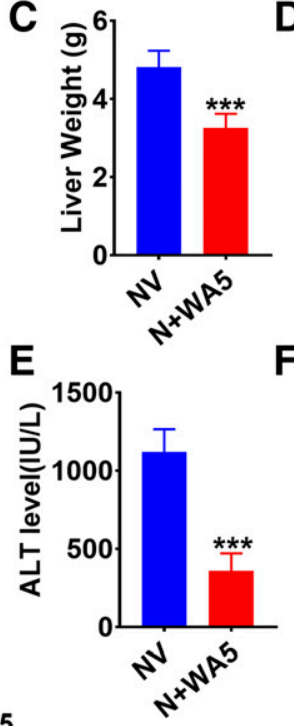

N+WA 5

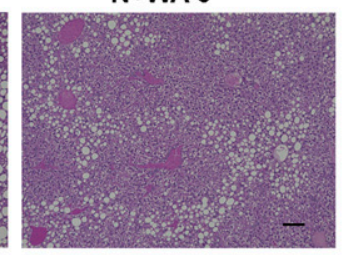

N+WA 5

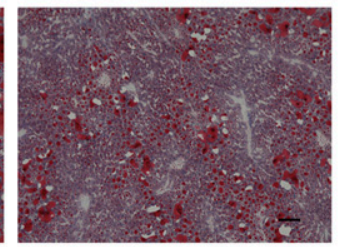

$\mathbf{F}$



$F$
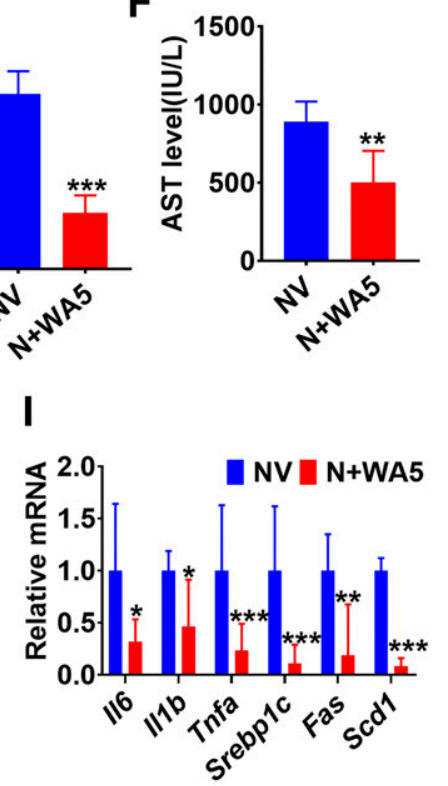

Fig. 11. WA improved HFD-induced liver injury in ob/ob mice and liver histology. (A) Experimental scheme for testing the therapeutic effect of WA in the HFD-induced NASH model. (B) Body weights. (C and D) liver weights (C) and liver indexes calculated by liver weight/body weight ratios (D). (E and F) Analysis of serum ALT (E) and AST (F) levels. (G and H) Representative histology pictures of H\&E staining (G) and Oil Red O staining (H) for HFD and WA-treated HFD livers. Scale bar, $100 \mu \mathrm{m}$. (I) Effect of WA on HFD-induced liver Il1b, Il6, Tnfa, Srebp1c, Fas, and $S c d 1$ mRNA levels. Data were presented as means \pm S.D. NV, mice fed the HFD-induced NASH diet (N) and treated with vehicle (V) for 12 weeks; N+WA5, mice fed the HFD-induced NASH diet and treated with $5 \mathrm{mg} / \mathrm{kg}$ WA for the last 4 weeks. $n=5$ mice for the HFD-fed vehicle groups and $n=8$ for WA treated HFD-fed ob/ob mice. ${ }^{*} P<0.05$; $* * P<0.01 ; * * * P<$ 0.005 compared with HFD group. Statistical differences were determined by the two-tailed $t$ test. dysregulation of oxidative stress and lowered ceramides that may be involved in the mechanism of NASH (Fig. 13). However, detailed mechanistic analysis required further studies.

The MCD diet-induced NASH model has been widely used for evaluating the pharmacological effects of drugs on NASH (Hebbard and George, 2011), and provides convenience due to its short-term experimental duration. By using this rapid short-term model, the effective dose of WA for NASH was found to be $5 \mathrm{mg} / \mathrm{kg}$, which both preventing and therapeutically reduced MCD diet-induced NASH. Compared with the MCD diet-induced lean NASH model, the HFD-induced NASH model more accurately reflects the human clinical NASH pathologies, including obesity, insulin resistance, and high serum triglycerides, in addition to the typical hepatic steatosis, inflammation, and fibrosis (Hebbard and George, 2011); thus, this model was chosen for further determining the effects of WA on NASH. In the obese NASH model, WA treatment improved NASH-associated pathologies, including hepatic steatosis, inflammation, and fibrosis, in a timedependent manner. Since HFD diet feeding causes hepatic steatosis and minor fibrosis after a 2-month diet feeding that gradually progress to advanced NASH (Ding et al., 2018), the effect of WA treatments for 12 weeks during the full course of HFD feeding would be mainly regarded as a preventive effect, whereas WA treatment for 8 and 4 weeks after the onset of
NASH would evaluate the therapeutic effects. In addition to WA's effect in improving NASH, WA also decreased HFDinduced body weight gain, in agreement with its known antiobesity activity (Lee et al., 2016). Whether the effect of WA is the result of its antiobesity effect or a direct effect on NASH in the present HFD-induced NASH model still requires further study. By using the NASH-promoting HFD, the current study extends the known pharmacological effects of WA to improving NASH, beyond its previously identifed roled in ameliorating obesity-accompanied hepatic steatosis associated with its antiobesity effect (Lee et al., 2016). However, weight loss caused by WA treatment could potentially decrease the HFD-induced NASH. Given that the body weight was sharply decreased upon WA treatment in the current HFD-induced fat NASH model as well as in the previous study (Lee et al., 2016), it would be difficult to separate the antiobesity effect as the cause of decreased NASH as opposed to a direct pharmacological effect of WA on NASH. However, the MCD-induced NASH mice show lean body weight instead of obesity, and the inhibitory effect of WA on MCD diet-induced NASH suggests that WA has a direct hepatoprotective effect in treating NASH independent of obesity, at least in this nonobese NASH model. As noted earlier, the present study provides evidence that WA potently improves MCD diet-induced NASH, a lean NASH mouse model, in which some hepatoprotective effects, such as the therapeutic effect of 
A
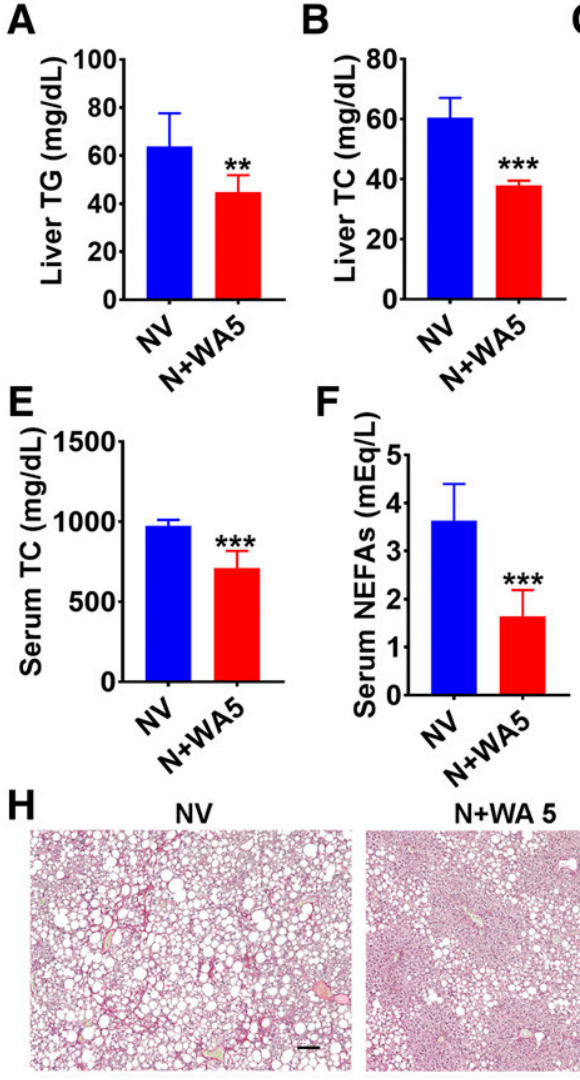

$F$

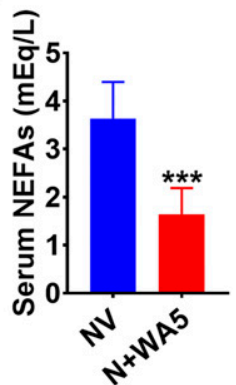

N+WA 5

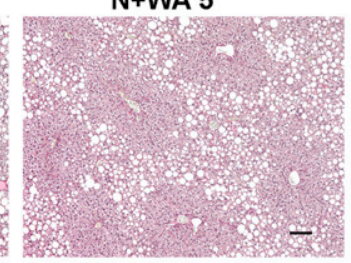

J

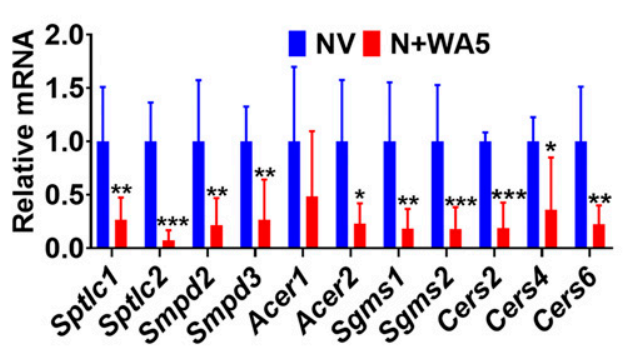

C
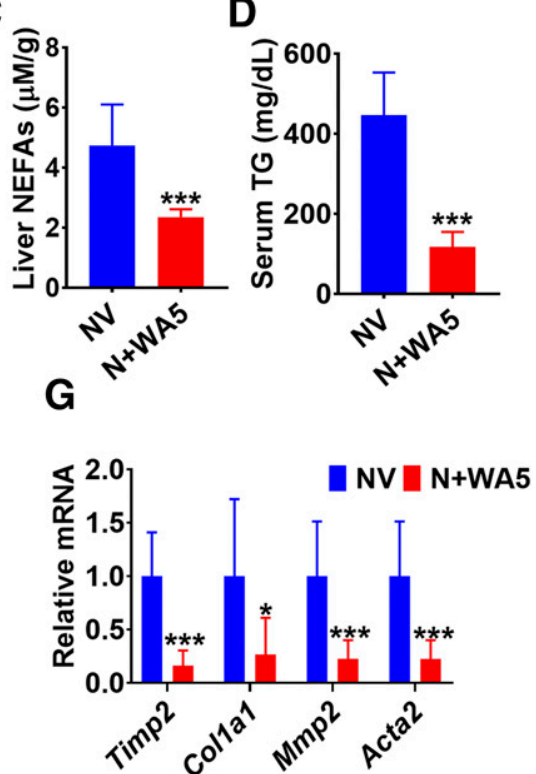

1



Fig. 12. WA treatment improved serum and liver biochemical parameters and reduced HFD-induced hepatic fibrosis and mRNAs associated with ER stress and ceramide biosynthesis and catabolism in WA-treated HFD-fed ob/ob mice. (A-F) Analysis of liver TG levels (A), TC levels (B), NEFA levels (C), serum TG (D), serum TC (E), and serum NEFA levels (F). (G) Hepatic fibrosis marker analysis of Timp2, Col1a1, Mmp2, and Acta2 mRNA levels. (H) Representative hepatic picrosirius red staining for HFD and WA-treated HFD livers. Scale bar, $100 \mu \mathrm{m}$. (I) Hepatic endoplasmic reticulum stress signaling marker analysis of Hspa 5 and Didt 3 mRNA levels. (J) Sptlc1, Sptlc2, Smpd2, Smpd3, Acer1, Acer2, Sgms1, Sgms2, Cers2, Cers4, and Cers6 mRNA levels. Data were presented as means \pm S.D. NV, mice fed the HFD-induced NASH diet $(\mathrm{N})$ and treated with vehicle (V) for 12 weeks. N+WA5, mice fed the HFD-induced NASH diet and treated with $5 \mathrm{mg} / \mathrm{kg}$ WA for the last 4 weeks $n=5$ mice for the HFD-fed vehicle groups and $n=8$ for WA treated HFD-fed ob/ob mice. $* P<0.05 ; * * P<0.01 ; * * * P<0.005$ compared with HFD group. Statistical differences were determined by the two-tailed $t$ test.
WA at the dose of $5 \mathrm{mg} / \mathrm{kg}$ on MCD-induced NASH and the preventive effect on MCD-induced NASH at doses of 1 and $2.5 \mathrm{mg} / \mathrm{kg}$, are independent of body weight change. Furthermore, WA could also markedly decrease MCD diet-induced increase of serum ALT and AST and increased liver weights prior to body weight changes after MCD diet feeding for 10 days. Similar to the present finding, it was reported that the hepatoprotective component glycyrrhizin also reduced MCD diet-induced NASH without significantly affecting the MCD diet-induced body weight loss (Yan et al., 2018). On the other hand, WA was reported to therapeutically improve acetaminophen-induced acute liver injury even when injected 1 hour after acetaminophen dosing (Jadeja et al., 2015), indicating that WA has a direct hepatoprotective effect. The current work, together with previous studies, suggests that WA could improve NASH, at least potentially due to its direct hepatoprotective activity, independent of its antiobesity effect.

WA was previously reported to act as a leptin sensitizer during obesity and was also shown to exert an antiobesity effect independent of leptin signaling, as WA still had an antiobesity effect in leptin-deficient mice (Lee et al., 2016).
Thus, the question arose whether the anti-NASH effect of WA was dependent on leptin signaling. Consistent with this previous study, WA still elicited a potent therapeutic effect in HFD-induced NASH in leptin-deficient ob/ob mice. Thus, WA has leptin-independent pharmacological activity in obesity and obesity-associated NASH.

In the present study, WA was found to reduce the accumulation of ceramides in serum accompanied by attenuation of HFD-induced upregulation of ceramides. WA also decreased hepatic oxidative stress and hepatic ER stress, which were correlated with its anti-NASH effects. Although all of these analyses provide potential hints for further mechanism exploration, the current study does not determine how WA directly affects oxidative stress signaling, ER stress signaling, ceramide signaling, or stress-treated ceramide signaling. In vitro studies are warranted to answer this question. Previous studies demonstrate that WA has antioxidant activity via activating NRF2 (Cassidy and Syed, 2016; Palliyaguru et al., 2016) and anti-inflammatory potential via directly inhibiting NLR family pyrin domain containing 3 (NLRP3) inflammasome activation (Kim et al., 2015; Dubey et al., 2018). Although WA attenuated NASH-induced NRF2 signaling at 


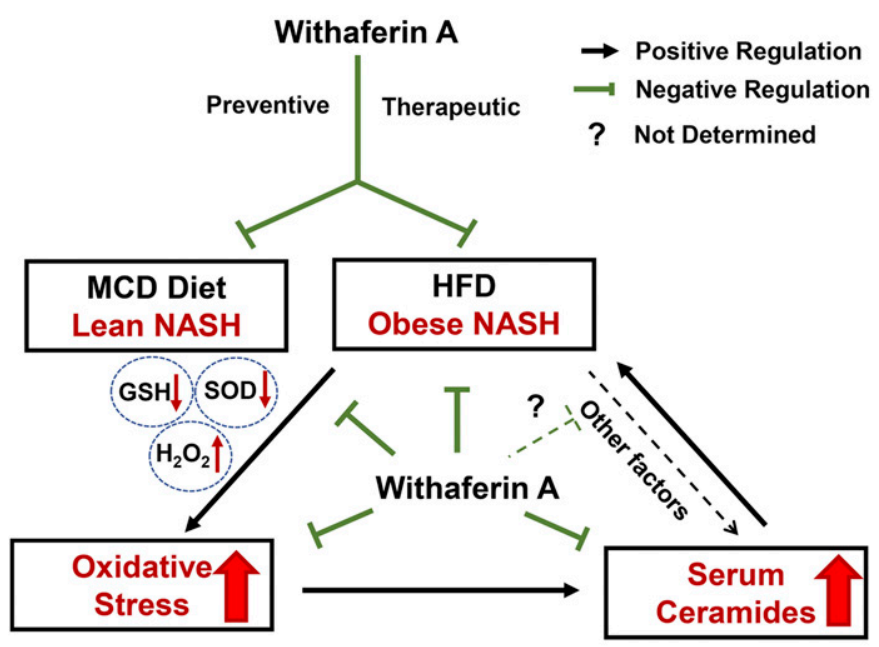

MCD: Methionine and Choline Deficient HFD: High-fat Diet

NASH: Non-alcoholic Steatohepatitis

Fig. 13. Summary of major findings and proposed mechanism. In the current study, WA was found to have both preventive and therapeutic effects in treating NASH as assessed by using two NASH models. MCD diet feeding induced weight loss and thus was called the "lean NASH" model, whereas HFD-induced obesity was referred to as the "obese NASH" model. HFD feeding induced oxidative stress, and oxidative stress could contribute to the increase in serum ceramide accumulation that was known to promote the progression of NASH. In the HFD-induced obese NASH model, WA alleviated HFD-induced oxidative stress as revealed by the decreased $\mathrm{H}_{2} \mathrm{O}_{2}$ and increased glutathione (GSH) and superoxide dismutase (SOD) levels. WA also significantly alleviated HFD-induced ceramide accumulation in serum. However, other factors could also contribute to ceramide accumulation in the HFD-induced NASH model, and how WA affects them still requires further study.

the terminal stages of the NASH model in the present study, which may be a result of its hepatoprotective effects, WA could activate NRF2 signaling in the early stages of NASH within 10 days of commencing MCD diet treatment, although WA did not activate hepatic NRF2 signaling after 1 week of commencing HFD feeding. Thus, in the current study, WA was able to modulate NRF2 signaling in a context-dependent manner.

NRF2 activation could improve glucose tolerance, suppress NASH and liver fibrosis, and attenuate liver cirrhosis (Wu et al., 2014; Sharma et al., 2017). This suggests that NRF2 could be an efficient target for anti-NASH therapy, consistent with the report that pharmacological activation of NRF2 by the potent NRF2 activator TBE-31 is able to ameliorate experimental NASH (Sharma et al., 2017). Multiple mechanisms are involved in the modulation of NRF2 signaling via interference with kelch-like ECH-associated protein 1-NRF2 interaction or other post-transcriptionally and/or post-translationally modulated mechanisms, such as endoplasmic reticulum acssociated protein degradation-associated E3 ubiquitin-protein ligase HRD1, glycogen synthase kinase 3, and $\beta$-transducin repeat-containing protein (Chowdhry et al., 2013; Wu et al., 2014; Hayes et al., 2015). Among these known NRF2-modulating mechanisms, WA was demonstrated to be an inducer of NRF2 signaling both in vitro and in vivo, and this effect is partially kelch-like ECH-associated protein 1-independent and in part dependent on the glycogen synthase kinase 3-associated modulation pathway, as in the acetaminophen-induced acute liver injury model in mice (Palliyaguru et al., 2016). Definitive determination of the mechanisms by which WA attenuates NASH, and whether they are dependent on NRF2 signaling or other pathways, such as NLRP3,requires further experimentation using NRF2 and NLRP3 knockout mice; these studies are beyond the scope of the current work.

In summary, by evaluating various NASH pathologic parameters in serum and livers in two well-defined NASH mouse models (MCD-induced NASH model and HFD-induced NASH model in wide-type mice), WA exhibits both preventive and therapeutic effects in improving NASH. WA also therapeutically decreases HFD-induced NASH in leptin-deficient ob/ob mice. The anti-NASH effect of WA is accompanied by lower hepatic oxidative stress, ER stress, and ceramide accumulation in serum. These data suggest that WA has potent anti-NASH effects independent of its antiobesity effect, as revealed in the nonobese MCD-induced NASH model, and is independent of its leptin-sensitizing effect, as found in an HFD-fed leptin-deficient ob/ob mouse NASH model. Although further studies to determine the exact mechanisms by which WA decreases NASH are still needed, these findings suggest that the herbal medicine-derived compound WA may be a therapeutic option for treating NASH, particularly that featuring ceramide accumulation, and would help repurpose the ancient drug WA with its novel application in treating NASH.

\section{Acknowledgments}

We thank Linda G. Byrd for submission and approval of the animal protocols; John Buckley, Linda G. Byrd, Ping Wang, and Yangliu Xia for help with animal experiments; and Chad N. Brocker, Jie Zhao, and Cen Xie for expert advice.

\section{Authorship Contributions}

Participated in research design: Yan, Patel, Gonzalez.

Conducted experiments: Patel, Yan, Kim, Dias, Krausz.

Performed data analysis: Patel, Yan, Dias.

Wrote or contributed to the writing of the manuscript: Yan, Patel, Gonzalez, Kimura.

\section{References}

Bikman BT and Summers SA (2011) Ceramides as modulators of cellular and wholebody metabolism. J Clin Invest 121:4222-4230.

Cassidy S and Syed BA (2016) Nonalcoholic steatohepatitis (NASH) drugs market. Nat Rev Drug Discov 15:745-746.

Chaurasia B and Summers SA (2015) Ceramides - lipotoxic inducers of metabolic disorders. Trends Endocrinol Metab 26:538-550.

Chowdhry S, Zhang Y, McMahon M, Sutherland C, Cuadrado A, and Hayes JD (2013) Nrf2 is controlled by two distinct $\beta$-TrCP recognition motifs in its Neh6 domain, one of which can be modulated by GSK-3 activity. Oncogene 32:3765-3781.

Ding ZM, Xiao Y, Wu X, Zou H, Yang S, Shen Y, Xu J, Workman HC, Usborne AL and Hua $H$ (2018) Progression and regression of hepatic lesions in a mouse model of NASH induced by dietary intervention and its implications in pharmacotherapy. Front Pharmacol 9:410.

Dubey S, Yoon H, Cohen MS, Nagarkatti P, Nagarkatti M, and Karan D (2018) Withaferin A associated differential regulation of inflammatory cytokines. Front Immunol 9:195.

Estes C, Razavi H, Loomba R, Younossi Z, and Sanyal AJ (2018) Modeling the epidemic of nonalcoholic fatty liver disease demonstrates an exponential increase in burden of disease. Hepatology 67:123-133.

Farrell GC and Larter CZ (2006) Nonalcoholic fatty liver disease: from steatosis to cirrhosis. Hepatology 43 (2 Suppl 1):S99-S112.

Flemming JA, Kim WR, Brosgart CL, and Terrault NA (2017) Reduction in liver transplant wait-listing in the era of direct-acting antiviral therapy. Hepatology 65:804-812.

Friedman SL, Neuschwander-Tetri BA, Rinella M, and Sanyal AJ (2018) Mechanisms of NAFLD development and therapeutic strategies. Nat Med 24:908-922.

Griffett K, Welch RD, Flaveny CA, Kolar GR, Neuschwander-Tetri BA, and Burris TP (2015) The LXR inverse agonist SR9238 suppresses fibrosis in a model of nonalcoholic steatohepatitis. Mol Metab 4:353-357.

Hayes JD, Chowdhry S, Dinkova-Kostova AT, and Sutherland C (2015) Dual regulation of transcription factor Nrf2 by Keap1 and by the combined actions of $\beta$-TrCP and GSK-3. Biochem Soc Trans 43:611-620.

Hebbard L and George J (2011) Animal models of nonalcoholic fatty liver disease. Nat Rev Gastroenterol Hepatol 8:35-44.

Heyninck K, Sabbe L, Chirumamilla CS, Szarc Vel Szic K, Vander Veken P, Lemmens KJA, Lahtela-Kakkonen M, Naulaerts S, Op de Beeck K, Laukens K, 
et al. (2016) Withaferin A induces heme oxygenase (HO-1) expression in endothelial cells via activation of the Keap1/Nrf2 pathway. Biochem Pharmacol 109:48-61.

Honda Y, Imajo K, Kato T, Kessoku T, Ogawa Y, Tomeno W, Kato S, Mawatari H, Fujita K, Yoneda M, et al. (2016) The selective SGLT2 inhibitor ipragliflozin has a therapeutic effect on nonalcoholic steatohepatitis in mice. PLoS One 11:e0146337.

Jadeja RN, Urrunaga NH, Dash S, Khurana S, and Saxena NK (2015) Withaferin-A reduces acetaminophen-induced liver injury in mice. Biochem Pharmacol 97:122-132

Javor ED, Cochran EK, Musso C, Young JR, Depaoli AM, and Gorden P (2005) Long term efficacy of leptin replacement in patients with generalized lipodystrophy. Diabetes 54:1994-2002.

Jiang C, Xie C, Li F, Zhang L, Nichols RG, Krausz KW, Cai J, Qi Y, Fang ZZ, Takahashi S, et al. (2015) Intestinal farnesoid $\mathrm{X}$ receptor signaling promotes nonalcoholic fatty liver disease. J Clin Invest 125:386-402.

Kasumov T, Li L, Li M, Gulshan K, Kirwan JP, Liu X, Previs S, Willard B, Smith JD, and McCullough A (2015) Ceramide as a mediator of non-alcoholic Fatty liver disease and associated atherosclerosis. PLoS One 10:e0126910.

Kim JE, Lee JY, Kang MJ, Jeong YJ, Choi JA, Oh SM, Lee KB, and Park JH (2015) Withaferin A inhibits helicobacter pylori-induced production of IL-1 $\beta$ in dendritic cells by regulating NF-кB and NLRP3 inflammasome activation. Immune Netw 15:269-277.

Kurek K, Piotrowska DM, Wiesiołek-Kurek P, Łukaszuk B, Chabowski A, Górski J, and Zendzian-Piotrowska M (2014) Inhibition of ceramide de novo synthesis reduces liver lipid accumulation in rats with nonalcoholic fatty liver disease. Liver Int 34:1074-1083.

Lee IC and Choi BY (2016) Withaferin-A--a natural anticancer agent with pleitropic mechanisms of action. Int $J$ Mol Sci 17:290.

Lee J, Liu J, Feng X, Salazar Hernández MA, Mucka P, Ibi D, Choi JW, and Ozcan U (2016) Withaferin A is a leptin sensitizer with strong antidiabetic properties in mice. Nat Med 22:1023-1032.

Longato L, Tong M, Wands JR, and de la Monte SM (2012) High fat diet induced hepatic steatosis and insulin resistance: role of dysregulated ceramide metabolism. Hepatol Res 42:412-427.

Michelotti GA, Machado MV, and Diehl AM (2013) NAFLD, NASH and liver cancer. Nat Rev Gastroenterol Hepatol 10:656-665.

Oseini AM and Sanyal AJ (2017) Therapies in non-alcoholic steatohepatitis (NASH). Liver Int 37 (Suppl 1):97-103.

Pagadala M, Kasumov T, McCullough AJ, Zein NN, and Kirwan JP (2012) Role of ceramides in nonalcoholic fatty liver disease. Trends Endocrinol Metab 23:365-371.

Palliyaguru DL, Chartoumpekis DV, Wakabayashi N, Skoko JJ, Yagishita Y, Singh SV, and Kensler TW (2016) Withaferin A induces Nrf2-dependent protection against liver injury: role of Keap1-independent mechanisms. Free Radic Biol Med 101:116-128.
Polyzos SA, Kountouras J, and Mantzoros CS (2015) Leptin in nonalcoholic fatty liver disease: a narrative review. Metabolism 64:60-78.

Scott N, McBryde E, Vickerman P, Martin NK, Stone J, Drummer H, and Hellard M (2015) The role of a hepatitis $C$ virus vaccine: modelling the benefits alongside direct-acting antiviral treatments. BMC Med 13:198.

Sharma RS, Harrison DJ, Kisielewski D, Cassidy DM, McNeilly AD, Gallagher JR, Walsh SV, Honda T, McCrimmon RJ, Dinkova-Kostova AT, et al. (2017) Experimental nonalcoholic steatohepatitis and liver fibrosis are ameliorated by pharmacologic activation of Nrf2 (NF-E2 p45-related factor 2). Cell Mol Gastroenterol Hepatol 5:367-398.

Thaiparambil JT, Bender L, Ganesh T, Kline E, Patel P, Liu Y, Tighiouart M, Vertino PM, Harvey RD, Garcia A, et al. (2011) Withaferin A inhibits breast cancer invasion and metastasis at sub-cytotoxic doses by inducing vimentin disassembly and serine 56 phosphorylation. Int $J$ Cancer 129:2744-2755.

Vanden Berghe W, Sabbe L, Kaileh M, Haegeman G, and Heyninck K (2012) Molecular insight in the multifunctional activities of Withaferin A. Biochem Pharmacol 84:1282-1291.

Vedi M and Sabina EP (2016) Assessment of hepatoprotective and nephroprotective potential of withaferin A on bromobenzene-induced injury in Swiss albino mice: possible involvement of mitochondrial dysfunction and inflammation. Cell Biol Toxicol 32:373-390.

Wong RJ, Aguilar M, Cheung R, Perumpail RB, Harrison SA, Younossi ZM, and Ahmed A (2015) Nonalcoholic steatohepatitis is the second leading etiology of liver disease among adults awaiting liver transplantation in the United States. Gastroenterology 148:547-555.

Wong RJ, Cheung R, and Ahmed A (2014) Nonalcoholic steatohepatitis is the most rapidly growing indication for liver transplantation in patients with hepatocellular carcinoma in the U.S. Hepatology 59:2188-2195.

Wu T, Zhao F, Gao B, Tan C, Yagishita N, Nakajima T, Wong PK, Chapman E, Fang D, and Zhang DD (2014) Hrd1 suppresses Nrf2-mediated cellular protection during liver cirrhosis. Genes Dev 28:708-722.

Xie C, Yagai T, Luo Y, Liang X, Chen T, Wang Q, Sun D, Zhao J, Ramakrishnan SK, Sun L, et al. (2017) Activation of intestinal hypoxia-inducible factor $2 \alpha$ during obesity contributes to hepatic steatosis. Nat Med 23:1298-1308.

Yan T, Wang H, Cao L, Wang Q, Takahashi S, Yagai T, Li G, Krausz KW, Wang G, Gonzalez FJ, et al. (2018) Glycyrrhizin alleviates nonalcoholic steatohepatitis via modulating bile acids and meta-inflammation. Drug Metab Dispos 46:1310-1319.

Address correspondence to: Frank J. Gonzalez, Laboratory of Metabolism, Center for Cancer Research, National Cancer Institute, National Institutes of Health, Bethesda, MD 20892. E-mail: gonzalef@mail.nih.gov 